
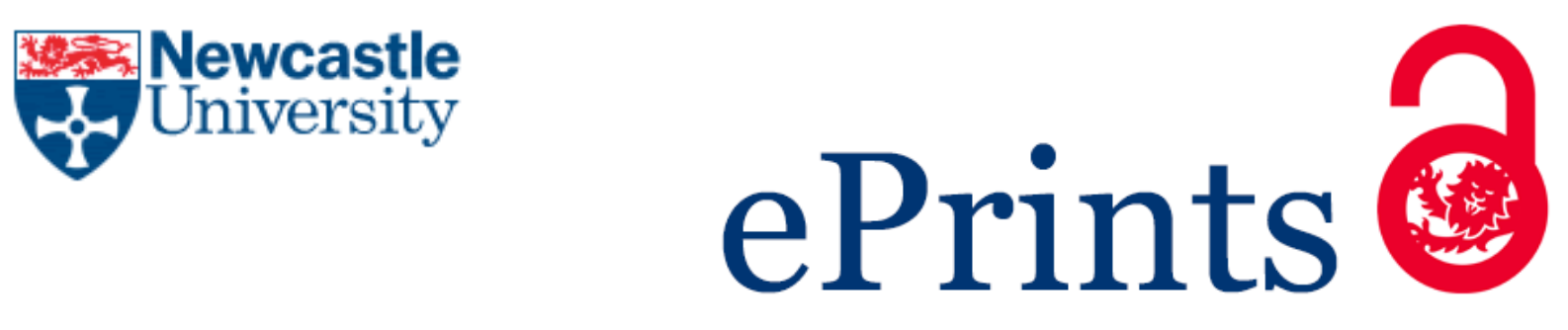

Weerasiri D, Barukh MC, Benatallah B, Sheng QZ, Ranjan R. A Taxonomy and Survey of Cloud Resource Orchestration Techniques. ACM Computing Surveys 2017, 50(2), 26.

\title{
Copyright:
}

(c) ACM, 2017. This is the author's version of the work. It is posted here by permission of ACM for your personal use. Not for redistribution. The definitive version was published in ACM Computing Surveys 50(2), (2017). https://doi.org/10.1145/3054177

DOI link to article:

https://doi.org/10.1145/3054177

Date deposited:

$22 / 08 / 2017$ 


\section{A Taxonomy and Survey of Cloud Resource Orchestration Techniques}

DENIS WEERASIRI, University of New South Wales, Australia

MOSHE CHAI BARUKH, University of New South Wales, Australia

BOUALEM BENATALLAH, University of New South Wales, Australia

QUAN Z. SHENG, University of Adelaide, Australia

RAJIV RANJAN, University of Newcastle, United Kingdom

Cloud services and applications prove indispensable amidst today's modern utility-based computing. The cloud has displayed disruptive and growing impact on everyday computing tasks. However, facilitating the orchestration of cloud resources in order to build such cloud services and applications is yet to unleash its entire magnitude of power. Accordingly, it is paramount to devise a unified and comprehensive analysis framework in order to accelerate fundamental understanding of cloud resource orchestration in terms of concepts, paradigms, languages, models and tools. This framework is essential to empower effective research, comprehension, comparison and selection of cloud resource orchestration models, languages, platforms and tools. This article provides such a comprehensive framework whilst analyzing the relevant state-of-the-art in cloud resource orchestration from a novel and holistic viewpoint.

Categories and Subject Descriptors: H.3.5 [Online Information Services]: Web-based services

General Terms: Methods, Techniques, Tools

Additional Key Words and Phrases: Cloud computing, resource orchestration, Service oriented architectures

ACM Reference Format:

Denis Weerasiri, Moshe Chai Barukh, Boualem Benatallah, Quan Z. Sheng and Rajiv Ranjan, 2016. A Taxonomy and Survey of Cloud Resource Orchestration Techniques. ACM Computing Surveys 49, 1, Article 0 (June 2016), 41 pages.

DOI : http://dx.doi.org/10.1145/0000000.0000000

\section{INTRODUCTION}

As economies undergo significant structural change, organisations are competitively compelled to leverage cloud computing to expand or contract their computing footprint based on variable demands for computing resources [Wang et al. 2012; Cui et al. 2013; Bahga and Madisetti 2013]. Typically, cloud providers enable virtualising three categories of resources - the "cloud computing stack" [Armbrust and et al. 2010]. These include, Software (e.g. user facing applications); Platform (e.g. development and runtime environments); and Infrastructure (e.g. storage, networking and hosting), [Ranjan et al. 2015; Satzger and et al. 2013; Weerasiri and Benatallah 2015]. Accordingly, cloud computing is evolving in the form of both public (deployed by IT organisations) and private clouds (deployed behind an enterprise firewall). A third option, a hybrid or federated cloud [Bahga and Madisetti 2013], draws computing resources from one or

Authors' addresses: D. Weerasiri, M. C. Barukh and B. Benatallah, School of Computer Science and Engineering, University of New South Wales, Australia; Q. Z. Sheng, School of Computer Science, The University of Adelaide, Australia; R. Ranjan, School of Computer Science, University of Newcastle, United Kingdom. Permission to make digital or hard copies of part or all of this work for personal or classroom use is granted without fee provided that copies are not made or distributed for profit or commercial advantage and that copies show this notice on the first page or initial screen of a display along with the full citation. Copyrights for components of this work owned by others than ACM must be honored. Abstracting with credit is permitted. To copy otherwise, to republish, to post on servers, to redistribute to lists, or to use any component of this work in other works requires prior specific permission and/or a fee. Permissions may be requested from Publications Dept., ACM, Inc., 2 Penn Plaza, Suite 701, New York, NY 10121-0701 USA, fax +1 (212) 869-0481, or permissions@acm.org.

(C) 2016 ACM 1539-9087/2016/06-ART0 $\$ 15.00$

DOI : http://dx.doi.org/10.1145/0000000.0000000 
more public clouds and one or more private clouds, combined at the behest of its users. A Gartner report [Gartner 2013] estimates that "nearly half of all large enterprises having cloud service deployments by the end of 2017".

However, there are crucial gaps in the cloud-enabled endeavor [Ranjan et al. 2015; Satzger and et al. 2013; Lu et al. 2013; Ranjan et al. 2013]. Modern orchestration frameworks like Puppet, Ubuntu Juju, Ansible, Amazon OpsWorks and Chef provide scripting-based languages for describing cloud configurations [Delaet et al. 2010]. Consequently, even sophisticated programmers are forced to understand various low-level cloud service Application Programming Interfaces (APIs), command line, and procedural programming constructs, to create and maintain complex resource configurations. This leads to a costly environment that lacks flexibility and is significantly more complex. It implies extensive programming effort, requires multiple and ongoing patches, and perpetuates closed-cloud solutions. This intensifies as the variety of cloud services and resource requirements increase. More specifically, with existing cloud delivery models, developing a new cloud-based solution often leads to uncontrollable fragmentation. This makes it very difficult to develop interoperable and portable cloud solutions. It also degrades performance as applications or workloads cannot be partitioned or migrated arbitrarily to another cloud when demand cycles increase. Moreover, cloud applications may have varying resource requirements during different phases of their life-cycle. Consequently, designing effective cloud orchestration techniques to cope with large-scale heterogeneous cloud environments remains a deeply challenging problem.

In this article, we propose a comprehensive analysis framework to effectively explore, assess, contrast and compare the variety of resource orchestration techniques. Previous surveys mostly focused on specific aspects and appear fragmented. Such as in: configuration management [Delaet et al. 2010]; monitoring [Bauman et al. 2015]; security and assurance [Ardagna et al. 2015; Huang and et al. 2015; Roy et al. 2015]; energy-efficiency [Mastelic et al. 2014]; adaptability [Singh and Chana 2015; Zhan et al. 2015a]; Quality of Service (QoS) and Service Level Agreements (SLAs) [Hani et al. 2015]; as well as software architectures for cloud-based systems [Chauhan et al. 2016] and interoperability concerns [Toosi et al. 2014; Lewis et al. 2013]. A preliminary overview of cloud orchestration tools are also presented in [Khoshkbarforoushha et al. 2016], and similarly an overview on cloud meta-models in [Bergmayr et al. 2015]. Nonetheless, there remains significant shortfalls in both the complementarity and breadth of understanding within cloud orchestration techniques. While previous efforts have produced encouraging and useful results, they are limited in scope with only a more 'broader' significance. In contrast, we present a holistic and comprehensive framework. We propose a taxonomy that is much more exhaustive with additional (sub-)dimensions that contribute to an 'in-depth' analysis over a mixture of techniques from both industry and academia. This is vital to understand the strengths and challenges, building blocks in terms of concepts, models, languages, techniques and tools and paves the way towards the next generation of cloud systems. To date this level of investigation has received little attention - this article aims to alleviate this gap.

We present an extensive survey in cloud resources orchestration. After introducing the necessary background (Section 2), we propose our taxonomy for understanding, analyzing and comparing cloud resource orchestration techniques (Section 3). We also discuss related work and the positioning of our taxonomy versus existing attempts. Our taxonomy sets out a framework of dimensions (resources, orchestration capabilities, user types, runtime environment and knowledge reuse), which we discuss progressively in Sections 4-8. We then apply the taxonomy to analyze a set of methodically chosen cloud resource orchestration tools and research prototypes; and identify several open research issues based on the technical gaps identified during the analysis (Section 9). Finally, we offer concluding remarks and directions for future study. 


\section{CLOUD RESOURCE ORCHESTRATION}

Consumers of cloud resources, human or software, typically have diverse requirements (e.g., storage capacity, access rules, etc.). Moreover, a single cloud resource often cannot provide all the necessary capabilities. Consider an HTTP server, application runtime and database, composed together to formulate a typical Web application deployment platform. The composition of dependent resources may require additional and complex configuration changes. For instance, a secured communication channel may be initialized between the application runtime and database by opening IP ports and enforcing access rules (e.g., firewall rules). Furthermore, deployed resources produce events (e.g., application server started, database server crashed), which need to be monitored so that necessary actions can be taken. To reason about this process, we introduce the notion of Cloud Resource Lifecycle, which aims to categorize orchestration tasks over the different phases in the typical lifespan of a cloud resource.

\subsection{Cloud Resource Lifecycle}

In much the same way practitioners have abstracted the lifecycle model, for example in the case of software engineering artifacts [Larman and Basili 2003], or Business Process Management (BPM) [Dumas et al. 2013] — we propose a similar lifecycle model suited for cloud resource artifacts. In essence, this model consists of the following phases: (1) Selection, consumers select required resources; (2) Configuration, resource description attributes are specified as well as relationships; (3) Deployment, cloud resources are instantiated; (4) Monitoring, resources are monitored to ensure they conform with Quality-of-Service (QoS) and Service Level Agreements (SLAs); and (5) Control, resources are dynamically (re-)configured to alleviate violations, or whenever there are changes in requirements.

At Section 5.1, we present a much more thorough description of these lifecycle phases together with relevant examples.

\subsection{Cloud Resource Orchestration Services and Operations}

To manage cloud resources over the lifecycle phases, various services and processes are used to: select, describe, configure, deploy, monitor and control cloud resources. We refer to the term Cloud Resource Orchestration to denote such processes and services. From the consumers' perspective, the function of orchestration systems are to bind resources and operations (e.g., deploy, monitor, scale-out), thereby providing an abstraction layer that shifts the focus from the underlying resource infrastructure, to available orchestration services and resource management [Wang et al. 2012]. Cloud resource orchestration systems implement a service-oriented model, enabling consumers to satisfy their application requirements by utilizing resources from cloud environments. In this manner, the overall goal of cloud resource orchestration is to ensure successful hosting and delivery of applications by meeting the QoS objectives of consumers.

In Fig. 1, we devise a reference architecture for cloud resource orchestration systems. In the following, we categorize processes, services, involved in cloud resource orchestration based on their functionalities vis-à-vis this reference model.

- Resource Provisioning Layer. Some services and tools merely offer the most basic operations to create, reconfigure and delete cloud resources. Such services and tools are built upon a resource description model - a meta-model that allow to describe resource configurations. For example, AWS Command Line Interface (CLI) [AWS 2013b] provides a range of provisioning services for every resource that they support. One such service offers operations (e.g., create, start, stop, delete, clone, attach storage volumes) to provision EC2 virtual machines [AWS 2013a]. 


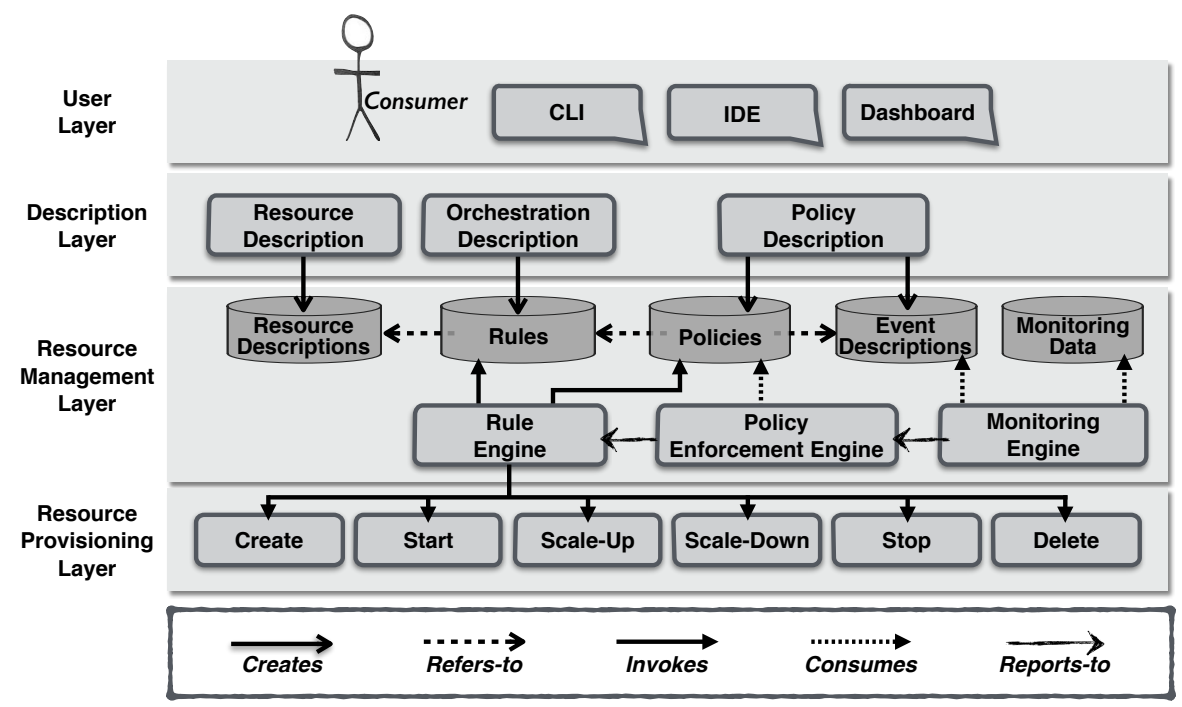

Fig. 1: Reference Architecture for Cloud Resource Orchestration

- Resource Management Layer. Effective automation of cloud resource management is imperative, as otherwise consumers are forced to manually build management logic over basic low-level operations offered by the Resource Provisioning Layer. For instance, automating a complex management task such as throughputbased Web application scaling in AWS requires: (i) a monitoring engine (e.g., AWS CloudWatch [CloudWatch 2013]); (ii) a policy enforcement engine (e.g., AWS Auto Scaling [Auto Scaling 2015]); and (iii) a rule engine (e.g., Opscode Chef [Sabharwal 2014]). The monitoring engine: collects throughput metrics from Web application servers; and thereby publishes events to a Policy Enforcement Engine (PEE). Based on the captured metrics, the PEE determines what decisions to make (e.g. replicate the Web application into multiple instances). The PEE invokes the rule engine to execute orchestration processes (e.g., clone, deploy and notify the HTTP load balancer about new instances). In some cases execution may be delegated to a process-engine, which coordinates the required scaling by leveraging operations at the Resource Provisioning Layer. Furthermore, services such as AWS Marketplace [Marketplace 2012], offer consumers to discover, create, curate and share knowledge about resource provisioning and management as reusable artifacts.

- Description Layer. This refers to languages and models to represent configuration, deployment, monitoring and control tasks of cloud resources. Typically as: (i) resource descriptions; (ii) orchestration processes/rules; and/or (iii) policies.

- Resource Descriptions define the configuration information of resources, as well as their relationships. For example, in AWS OpsWorks, a collection of Web application components (e.g., database, application engine, HTTP load balancer) and relationships can be defined via JSON notation [Amazon 2015; Rosner 2013].

- Orchestration Descriptions describes the 'behavioral' aspects, (i.e. control and re-configuration) of the cloud resources. There are declarative approaches (e.g. CloudFormation provided by AWS, or Heat provided by OpenStack); or imperative approaches that are based on processes (i.e. workflows). In some cases, consumers explicitly define deployment and/or configuration rules. For example, AWS OpsWorks provides a language with a set of pre-defined lifecycle events (e.g., 
setup, configure, deploy, undeploy, shutdown), which may be associated to orchestration actions. Other approaches, often based on workflows, requires no explicit rules, albeit the Rule-Engine may delegate to a process-engine.

- Policy Descriptions endow resources with dynamic control behaviors. For example, defining load-based policies to scale Web applications. Such a policy allows to instantiate new application engines when the average CPU utilization exceeds 95\% and stop application engines when their average CPU load falls below $40 \%$.

- User Layer. Cloud resource consumers (e.g., system admin or app developers) may interact with services of the other layers. Command Line Interfaces (CLIs), Software Development packages (SDKs), APIs and Integrated Development Environments (IDEs) (e.g., AWS CLI, AWS Java SDK, AWS REST API and VisualOps) expose services to manipulate cloud resource descriptions, orchestration rules and policies [AWS 2013b; Amazon Web Services 2015c; Amazon Web Services 2015d; VisualOps 2015]. Dashboards allows for interactions using humanfriendly abstractions. For instance, Amazon CloudWatch [CloudWatch 2013]) represents monitoring Data using format pre-built UI components and charts.

\section{CLOUD RESOURCE ORCHESTRATION TAXONOMY}

To offer systematic analysis, we introduce our taxonomy as depicted in Fig. 2. We identify the main dimensions and common building blocks that characterize cloud resource orchestration techniques, and available solutions. The taxonomy is a result of our own research efforts, experiences from industry, extensive literature reviews in related areas, as well as experiments with various services and tools.

Earlier, we discussed what is meant by "cloud resources orchestration". Based on the identified taxonomy, we now focus how such orchestration can be described, deployed and provisioned - independently of specific technologies or target solutions. Accordingly, we identify five main dimensions to characterize cloud resource orchestration techniques, which in turn are split into various sub-dimensions. A portion of this analysis also requires figuring out how a specific dimension may affect others, (e.g., which resource access methods are suitable for which user categories).

(1) Resources. This dimension identifies the formalisms that are offered for representing cloud resources. We further consider what resources are supported, how resources are modeled, represented and accessed (refer to Section 4).

(2) Orchestration Capabilities. This consist of actions and processes to manage orchestration tasks. We further divide this dimension into sub-dimensions and look at orchestration actions, paradigms, automation strategies and theoretical foundations (refer to Section 5). (We summarize cross-cutting concerns at Appendix B.)

(3) User Type. In our analysis, we identified three categories of users who have different roles in managing cloud resources (refer to Section 6).

(4) Runtime Environment. We identify three relevant sub-dimensions: (i) Virtualization technique; (ii) Execution model; and (iii) Target environment.

Virtualization technique refers to how physical resources are abstracted to simplify their consumption. The Execution model refers to how cloud resources are deployed, monitored and controlled in a distributed environment. Target environment identifies different deployment models such as public, private and federated/hybrid cloud environments (refer to Section 7).

(5) Knowledge Reuse. Productivity may be further enhanced through supportive reuse capabilities of existing orchestration knowledge. Users may implement and share orchestration knowledge as reusable software artifacts (e.g., resource descriptions, orchestration rules). We identify two sub-dimensions of knowledge reuse: (i) Reused Artifact; and (ii) Reuse Technique (refer to Section 8). 


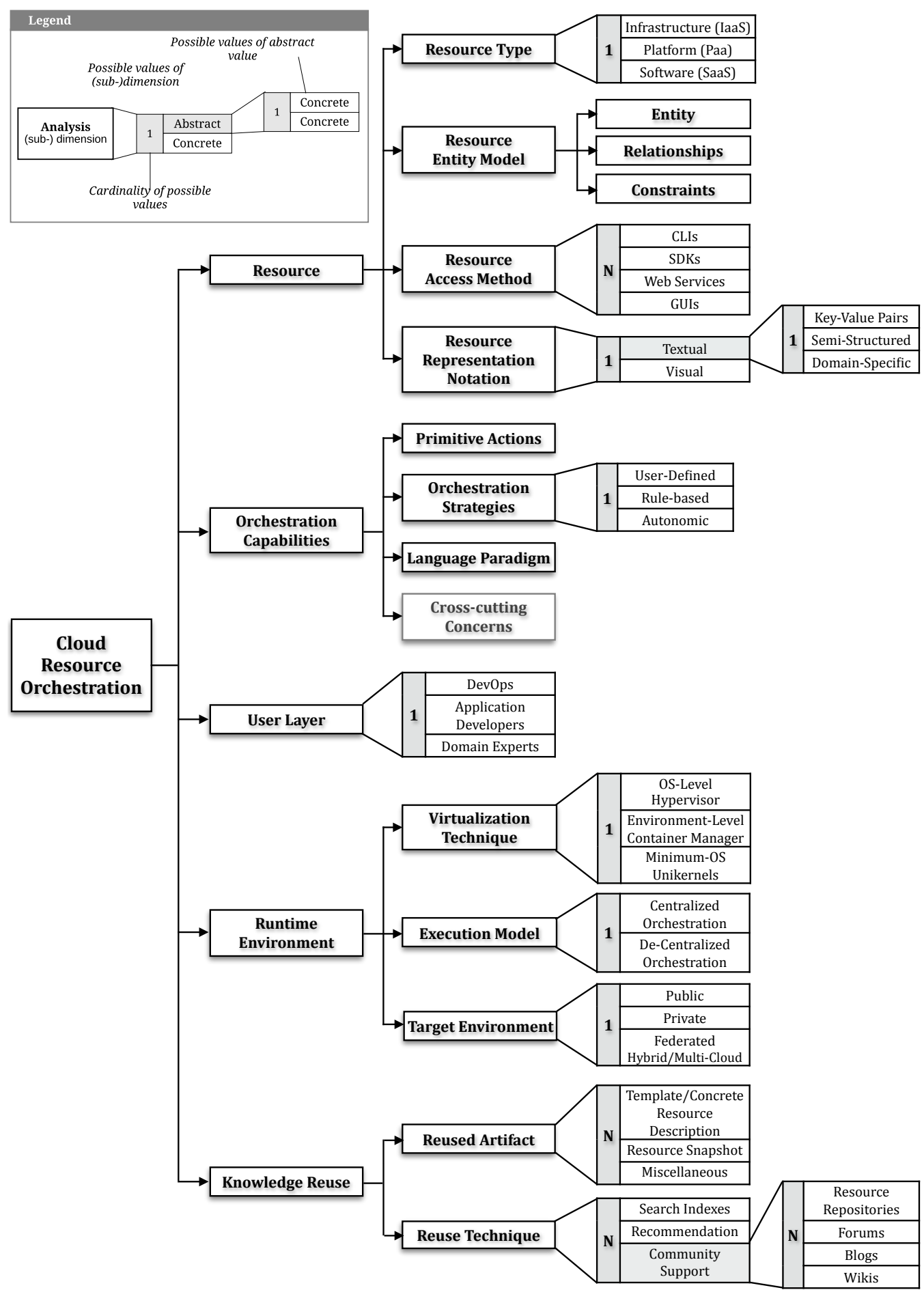

Fig. 2: A Taxonomy in Cloud Resource Orchestration 


\subsection{Related Work: Positioning versus Existing Taxonomies}

A holistic taxonomy and a comprehensive framework for in-depth analysis has not yet been addressed in a manner comparable to the approach presented in this paper:

High-level cloud computing taxonomies have been defined by OpenCrowd [OpenCrowd 2010], Rimal et al. [Rimal et al. 2009], and leading industry vendors (Intel [Intel-Corporation 2015], Oracle [Oracle-Corporation 2011], and Cisco [Cisco-SystemsInc. 2011]). Albeit, the applicability of cloud taxonomies proposed by industry vendors thus far, is limited to understanding their: (i) product strategies and business capabilities; (ii) key cloud product suppliers; and (iii) cloud computing strategies. In contrast our taxonomy addresses technical resource orchestration concerns for all resource types (IaaS, PaaS, and SaaS) across all orchestration layers (Resource Provisioning, Resource Management, Description and User Layers), refer to Fig. 1.

Forrester [Ried et al. 2010] introduced a market-oriented taxonomy of cloud computing. Similarly, early cloud computing taxonomies proposed by Hoefer et al. [Hoefer and Karagiannis 2010] and Laird [Laird 2008] took a very simplistic view. These taxonomies once again failed to incorporate technical concerns and dependencies across multiple orchestration layers which is clearly a novel contribution of our paper.

Chana et al. [Singh and Chana 2016] devised an autonomic (self-* properties) resource management taxonomy that covers aspects related to runtime QoS management of cloud-hosted applications, including: (i) monitoring; (ii) fault-tolerance; (iii) workload consolidation; and (iv) scheduling objective function and QoS metrics. On the other hand, Tossi et al. [Toosi et al. 2014] presented cloud resource management with a focus on application portability across federated public/private cloud data centers. Similar to [Singh and Chana 2016], the taxonomy presented in [Toosi et al. 2014] is limited to QoS and SLA management aspects (autonomic scheduling, portability, monitoring, security, cross-cloud communication). Similarly, Zhang et al. [Zhan et al. 2015b] presented a taxonomy to help in understanding and analyzing the application of Evolutionary Computing (EC) techniques for formulating application scheduling heuristics (i.e., Orchestration Strategies). Although, the taxonomies presented in [Singh and Chana 2016; Toosi et al. 2014; Zhan et al. 2015b] are very detailed from the perspective of application QoS management (which evidently draws parallel with Runtime Environment dimension and Orchestration Strategies sub-dimension in Fig. 2), they failed to cover other important dimensions and sub-dimensions related to holistic cloud resource orchestration process, such as User Layer, Knowledge Reuse, Resource Access Method, Resource Representation Notation, Language Paradigm, etc. Refer to Fig. 2.

Different standardization bodies have also proposed reference cloud computing architectures including NIST [Liu et al. 2011c]; Distributed Management Task Force (DMTF) [DMTF 2010]; Cloud Security Alliance (CSA) [CSA 2011]; and Internet Engineering Task Force (IETF) [Khasnabish et al. 2011]. Although these reference architectures aids in general understanding of cloud computing model, they are not based on a systematic taxonomy. They hence lack in-depth technical concepts (i.e., dimensions and sub-dimensions) required to understand the holistic nature of cloud resource orchestration processes, which is clearly a novel contribution of our proposed taxonomy.

Several recent papers [Beloglazov et al. 2011; Shuja et al. 2014; Hameed et al. 2014] have introduced taxonomies related to energy-efficient scheduling (an instance of $\mathrm{Or}$ chestration Strategy) of applications on cloud data centers. Our taxonomy does not explicitly focus on energy efficiency, rather proposes a holistic taxonomy that covers end-to-end lifecycle aspects of cloud resource orchestration processes.

Attempts [Moscato and et al. 2011; Fang et al. 2015; Zhang et al. 2012b; Dukaric and Juric 2013] to define an ontological model to understand basic cloud resource types, their entity models, dependencies, and orchestration operations has been un- 
dertaken by several authors. For example, EU mOSAIC's project [Moscato and et al. 2011] proposed an ontology that lets application developers understand basic resource types, their entity models, dependencies and configurations in a multi-cloud environments. On the other hand, Pahl et al. [Fang et al. 2015] proposed the SAMOS ontology that models entities (concepts), supported orchestration operations across SaaS, PaaS, and IaaS layers, and entity-to-entity relationships. Similarly, Juric et al. [Dukaric and Juric 2013] propose an ontological taxonomy to characterize IaaS resource types and related orchestration operations. The taxonomy is structured around seven orchestration layers: core service layer, support layer, value-added services, control layer, management layer, security layer and resource abstraction. Finally, EU Cloud4SOA project [Kamateri et al. 2013] proposed an ontological model to express relationship and dependencies between PaaS offerings across different cloud providers that share the same virtualization technology. Although these ontological models identify the necessary information related to cloud resource entity models and their relationships with other entities, in contrast to our work these ontological taxonomy models lack the focus on other important taxonomy dimensions (Orchestration Capabilities, User Layer, Knowledge Reuse, Runtime Environment), which are highly mandatory for understanding the holistic nature of cloud orchestration process.

In another strand of cloud computing research, authors have developed taxonomy [Fatema et al. 2014; Alhamazani et al. 2015] of cloud monitoring (a type of runtime orchestration operation) techniques and tools. However, these taxonomies have considered the monitoring problem in silos - and have failed to cover other important resource orchestration dimensions, such as Orchestration Capabilities, Resource, RunTime Environment, User Layer, and Knowledge Reuse. We present an encompassing general-purpose framework focused on all essential, interdependent dimensions of the cloud resource orchestration process.

\section{RESOURCES}

\subsection{Resource Types}

Cloud providers enable virtualizion through three categories of resources, namely: namely: Infrastructure, Platform, and Software -as-a-Service.

- Infrastructure. Infrastructure resources represent processing, storage, network and hosting environments [Bittman 2011; Armbrust and et al. 2010; Wang et al. 2012; Thrash 2010; Ranjan et al. 2015]. Providers include: VMWare vSphere, OpenStack, AWS EC2 CLI, Google Cloud Platform, OpenNebula, Eucalyptus, CohesiveFT, CloudStack and Rackspace [Lowe 2011; OpenStack.org 2015a; AWS 2013a; Platform 2015; Networks 2016; Project 2016; Development 2016; CloudStack 2016; Cali 2013]. However, some providers do not support all types of infrastructure resources. For example, Rackspace allows to describe virtual machines (VM), associate storage volumes and create communication channels among VMs. On the other hand, Juju [Ubuntu 2013] only supports provisioning Ubuntu based VMs and does not support storage or network resources.

- Platform. Platform resources provide software development tools, middleware, SDKs and/or APIs. It also supports run-time environments, such as content delivery networks, mobile application run-times and Big-data platforms; all which facilitate coding and deploying software resources. Providers include, AWS OpsWorks, AWS CloudFormation, Google App Engine, Cloud Foundry, Ubuntu Juju, Puppet, Chef, Ansible, Heroku, EngineYard, CloudBees and nitrous.io [Rosner 2013; Amazon 2011; Google 2015b; Cloud-Foundry 2016; Ubuntu 2013; Middleton et al. 2013; Engine Yard 2016; CloudBees 2016; Nitrous 2013; Labs 2015a]. For example, Heroku provides language runtimes such as Java, Ruby and Node.js. 
- Software. Software resources are applications (i.e., Web or mobile) for servicebased software delivery model [Cusumano 2010; Ranjan et al. 2015]. For example, Salesforce. com $^{1}$ provides pay-per-use Customer Relationship Management (CRM). Software resources are the most abundant type of resources compared to Platform or Infrastructure resources [Gartner 2014].

\subsection{Resource Entity Model}

We propose the notion of Resource Entity Model to represent the structure of cloud resources and their relationships. This implies a high-level resource model, which we represent as a graph, whose nodes and edges correspond to cloud Resource Entities and their Relationships respectively [Chen 1976]; as well as any related Constraints.

4.2.1. Resource Entity Types. An entity type describes properties of cloud resources via a set of attributes (e.g., key-value pairs), and as such characterizes the possible runtime instances of the resource type. For example, a VM provided by AWS EC2 [Services 2015a], has attributes such as number of CPU cores, storage capacity, memory capacity, operating system and access rules. System administrators specify values for before deploying, and once deployed it may include additional attributes like instance ID, public IP address, and launched time to represent the runtime state.

Resource entities can be further categorized as Elementary or Composite. An elementary resource does not rely on any other resources, while acting as the primary building blocks of composite resources. A composite resource is an umbrella structure that brings together other elementary and composite resources to describe a larger cloud resource. For example, an E-Learning platform that consists of an artifact management service and student identity management service to support 100 students.

Resource entities may be described at various levels of granularity. For example, Puppet [Kanies 2006] orchestrates resources within a single physical or virtual machine. Primary resource entities are thus fine-grained such as, file, sshkey and package [Labs 2015d]. Coarse-grained resources such as application engines (e.g., Node.js runtime) are composed of fine-grained resources. In contrast, Juju [Ubuntu 2013] is primarily dedicated to orchestrating resources deployed across multiple machines. Juju provides resource entity types called Charms, which represent high-level services (e.g., Node.js runtimes, Hadoop clusters) as primary resource entities.

Most orchestration techniques only support describing resources of a specific provider [Konstantinou and et al. 2009; Wittern et al. 2014; AWS 2013b]. On the other hand, others such as TOSCA, ModaClouds and CloudBase provide cross-provider cater for Resource Entities that are portable across different providers [Binz and et al. 2013; Ardagna and et al. 2012; Weerasiri et al. 2015a]. Orchestration techniques that support cross-provider resources (e.g., ComputeService in JCloud) are often intended for configuration and management of federated or hybrid cloud resources [Foundation 2014b; Elmroth and Larsson 2009; Villegas and et al. 2012].

4.2.2. Resource Relationships. A Relationship denotes a link between two Resource Entities. The relationship constructs can be further annotated with key-value pairs, in order to describe the properties of the respective relationship.

In circumstances where an orchestration technique does not support the explicit descriptions of relationships, composite resources may in fact become inconsistent when orchestrating two related component resources. Consider a Web application, such as LAMP suite (i.e., a Linux software stack, including an Apache HTTP server, MySQL database server and PHP application engine) [Lawton 2005]. When the associated

$\overline{{ }^{1} \mathrm{http} / / / \mathrm{www}}$.salesforce.com 
database server is migrated to a new IP address, this means that the relevant configuration attributes held at the application engine should also be updated. This is required in order to maintain successful communication between the application engine and database server. However, if the orchestration technique does not support explicit relationships, this implies system administrators may need to manually update the relevant attributes (or employ other 3rd-party tools such as shell scripts). These alternatives are error-prone and may also cause unnecessary overheads.

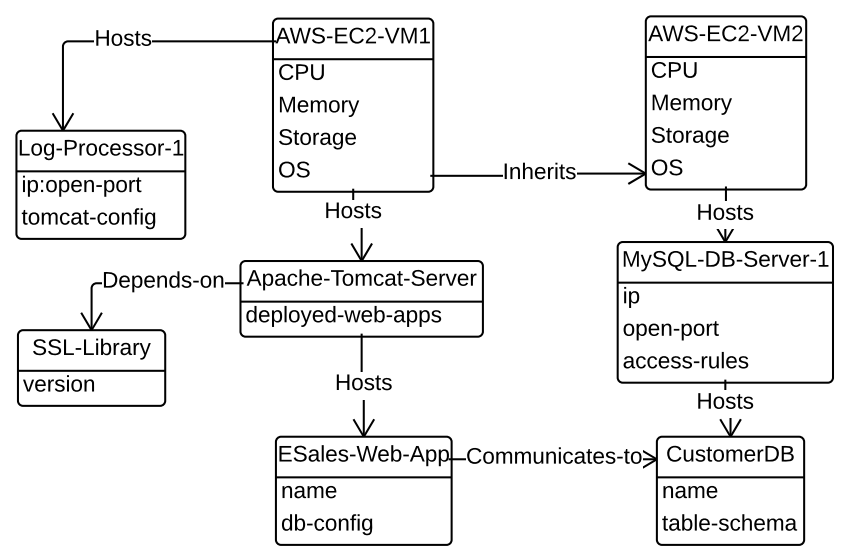

Fig. 3: Resource Entities and Relationships of a Web application

Relationships are established between a provider and consumer resource entities, where the provider offers some type of capability for the consumer. Fig. 3 exemplifies relationships of a typical Web application named ESales-Web-App with other resources. This Web application is "hosted" in Apache-Tomcat-Server and "communicates" data provided by CustomerDB, which is "hosted" in MySQL-DB-Server-1. Apache-Tomcat-Server and MySQL-DB-Server-1 are "hosted" in AWS-EC2-VM1 and AWS-EC2-VM2 respectively.

We identify the following types of relationships between cloud resources:

(1) Communication Relationship. Denotes the exchange data. For example, TOSCA 1.0 [OASIS 2013] provides a relationship type called ConnectsTo, e.g, between an application and its associated database, (refer to Fig. 4). TOSCA 1.0 thereby, interprets description attributes (e.g., communication protocol) of the relationship; and constructs a channel between the relevant resources.

\begin{tabular}{|l|l|l|}
\hline ESales-Web-App & Communicates-to $>$ & $\begin{array}{l}\text { CustomerDB } \\
\text { name } \\
\text { db-config }\end{array}$ \\
& $\begin{array}{l}\text { name } \\
\text { table-schema }\end{array}$ \\
\hline
\end{tabular}

Fig. 4: Communication Relationship between a Web Application and Database

(2) Dependency Relationship. Associate a given resource with other supporting resources that are required for successful operation. For example, a Web application server depends on a Secure Socket Layer (SSL) library (e.g., OpenSSL) to encrypt and communicate data with other resources, such as database server (refer to Fig. 5). TOSCA 1.0 provides a relationship type called DependsOn. In Ubuntu Juju [Ubuntu 2013], relationships are described as resource attributes that specify whether a given resource provides or requires a particular capability to/from 
another resource. For instance, MySQL DB provides a data source; whereas a Web application requires a data source). System administrators are able to create these relationships during deployment.

\begin{tabular}{|c|c|c|}
\hline Apache-Tomcat-Server & \multirow{2}{*}{-Depends-on $>$} & SSL-Library \\
\hline deployed-web-apps & & version \\
\hline
\end{tabular}

Fig. 5: Apache-Tomcat-Server depends on SSL-Library

(3) Inheritance Relationship. Denotes when the provider's attribute values are inherited by the consumer. However, the consumer resource is permitted to override the inherited attribute values to enable customizations. In other words, inheritance relationships are a convenient way of configuring attributes of a resource entity by reusing attribute values of another resource entity. For example, to describe a new Web application, which is to be installed on Apache Web server and Ubuntu Operating System: An application developer may simply inherit an existing Web Application resource with a similar configuration - all relevant attributes are inherited (refer to Fig. 6). Similarly, in Fig. 3, an Inheritance relationship is set up from AWS-EC2-VM1 to AWS-EC2-VM2. This relationship enforces VM2 to include the same version of operating system described VM1.

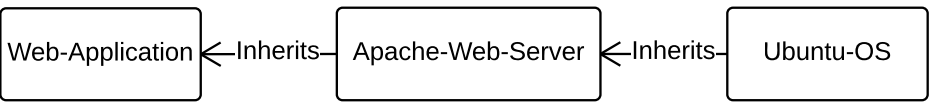

Fig. 6: Inheritance Relationships between Docker Images

(4) Containment Relationship. Denotes a parent-child relationship in which orchestration actions on a parent automatically trigger actions on all children. In practice, containment relationships are used to conveniently orchestrate a set of related resource entities together. For example, AWS OpsWorks [Rosner 2013] provides a resource entity type called Stack. It represents a Web application and may contain a set of child entities that are required to build a Web application, such as Apache Tomcat Server and MSQL database (refer to Fig. 7). When the Stack entity is deleted, consequently all children are deleted automatically.

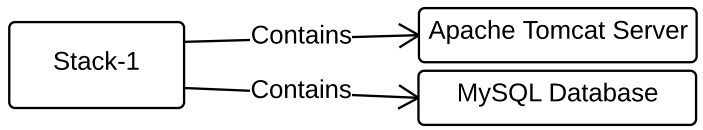

Fig. 7: Containment Relationships within an OpsWorks Stack

(5) Hosting Relationship. Enforces deployment of the consumer within the provider resource. This is useful when multiple component resources need to be deployed within a single component resource. For example, a log-file processor and an application server need to be deployed within a single VM, as the log-file processor needs the local file system access to read application server logs (see Fig. 8 and 3). For example, Ubuntu Juju [Ubuntu 2013] enables users to specify the infrastructure resource provider (e.g., AWS, HP-Cloud, Windows Azure), that will be used to deploy platform resources. Similarly, TOSCA 1.0 [OASIS 2013] supports a hosting relationship called HostedIn, where the deployment engine interprets the relationships and resolves which resource is to be hosted into which resource. 


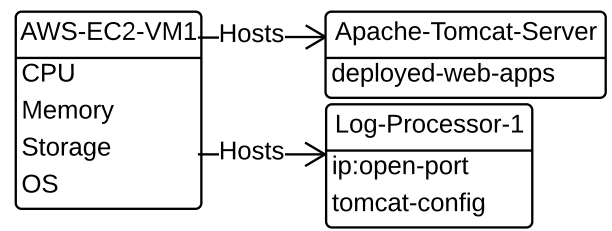

Fig. 8: Web application server and Log processor, hosted in one VM

4.2.3. Constraints. In some circumstances, it may be necessary to restrict the type of resource entities or relationships. For instance, AWS does not allow creating EC2 VMs with arbitrary amounts of CPU, memory and storage. Instead, a set of VM types (e.g., micro, medium, large) are provided, which are optimized for different use cases (e.g., low-traffic Web applications, large databases) [Amazon Web Services 2015a]. From a non-technical standpoint, having a constrained set of VM types allows providers to maintain simpler billing policies. Similarly, constraints may restrict resource relationships. For example, an AWS EC2 VM must be configured with a 64-bit CPU in order to install a 64-bit OS on the particular AWS-EC2 VM.

For resource entities, constraints are specified by restricting the possible values of attributes. For relationships, constraints are specified using: cardinality and participants. Consider a Ubuntu OS installed within an AWS EC2 VM. Here a one-to-one relationship exists, given that neither the Ubuntu OS can exist within more than one VM; nor multiple OSs can be installed with a VM simultaneously. Whereas, one-tomany relationships may exists between a cluster of HTTP Web servers and their load balancer. Sometimes the cardinality may be arbitrary, e.g., Ubuntu Juju allows users to specify maximum and minimum numbers of consumers (e.g., Web application) that may create relationships with a provider (e.g., Web application engine) [Juju 2015b].

We also identify two further orthogonal sub-categories of the Participants relationship, namely, (i) Inter-Vendor; and (ii) Vendor-Specific relationships.

(a) Inter-Vendor relationships. In some cases, relationships are permitted between two participating resources from different orchestration vendors. For example, Google App Engine [Google 2015b] allows users to deploy applications on a Google Compute VM or as a Docker container. DevOps are therefore allowed to associate infrastructure and platform resources across these different vendors.

(b) Vendor-Specific relationships. Some providers do not permit relationships between other vendors; and in some cases even restrict between different resource types (i.e., infrastructure, platform and software). For example, DotCloud [dotCloud 2015] only permits a composition of platform resources (e.g., databases, application engines) on top of a specific infrastructure resource (e.g., AWS EC2). DevOps are therefore not allowed to configure or reconfigure the infrastructure resources. On the other hand, CA-AppLogic allows users to specify which platforms resources are to be deployed on which infrastructure resources.

Furthermore, standards such as OCCI [Metsch et al. 2010] may be imposed as a means for providing semantics over resources, defining the type of a given entity, describing interdependencies between various entities, and defining operating characteristics on them. The goal being to facilitate extensibility and interoperability.

\subsection{Resource Access}

Over the years, software interfaces have evolved offering various designs to cater for the different capabilities of diverse users. Similarly, in the context of cloud orchestration, we have identified four types of interfaces [Khoshkbarforoushha et al. 2016]: 
4.3.1. Command Line Interfaces (CLIS). CLIs offer a fixed set of commands each of which includes a specified set of input, output and error parameters. For example, the AWS CLI [AWS 2013b] suite allows users to configure, deploy and control cloud resources; such as VMs, data storage and load balancers. As shown in Code 1, the "run-instances" command allows DevOps to deploy a specified number of VMs in the AWS public cloud infrastructure. The input parameters describes the VM configuration and the number of VMs to be launched in terms of key-value pairs. The output of the command execution is a JSON-based description of the resultant deployment.

Code 1: AWS CLI command to deploy VMs

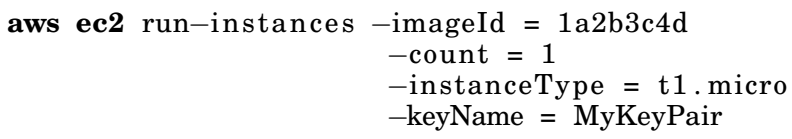

4.3.2. Software Development Kits (SDKs). AWS provides SDKs for a wide range of languages (e.g., Java, PHP, .NET and Ruby). For example, DevOps may download the Java-based SDK and thereby write Java applications to configure and deploy cloud resources in AWS cloud infrastructure (refer to Code 2). While CLIs are intended for system administrators with less application development skills, SDKs are intended for those with expertise in particular programming languages.

Code 2: Java Syntax in AWS SDK to deploy a VM

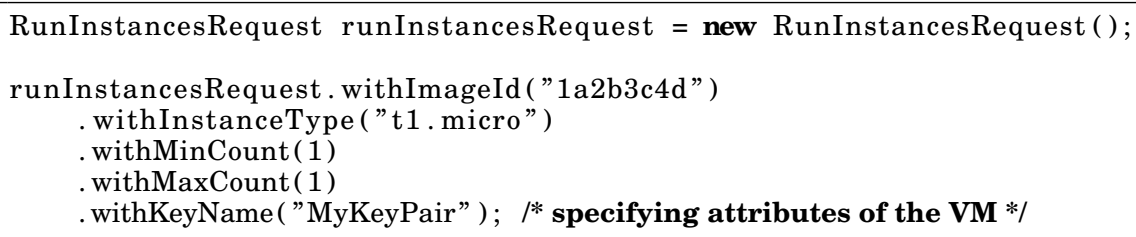

4.3.3. Application Programming Interfaces (APIs). Compared to SDKs, APIs provide language independent interfaces for orchestration capabilities that can be accessed by software applications, typically over HTTP. For example, Rackspace provides a RESTful API [Rackspace 2015] to configure, deploy and control cloud resources such as VMs, load balancers and databases.

4.3.4. Graphical User Interfaces (GUIs). GUIs comprise visual constructs to interact with orchestration services. For example, StackEngine, Panamax and Shipyard provide Web based GUIs to configure, deploy, monitor and replicate Docker containers [StackEngine 2015; CenturyLink 2015; shipyard 2015]. CA-AppLogic provides a desktop based GUI to manage software appliances in a private cloud infrastructure [AppLogic 2015]. Some other advanced GUIs, such as Puppet Enterprise Console and VisualOps provide dashboards which generate reports such as bar charts and maps (e.g., visualize the number of failed and running VMs during past 30 days) [Labs 2015c; VisualOps 2015].

\subsection{Resource Representation Notation}

Notations for representing resources and their relationships may consist of textual and/or visual constructs. We identified three classes, namely: textual, visual, and hybrid (a mix of textual and visual) notations. 
4.4.1. Textual notations. We distinguish between three variations of textual notations:

(1) Key-value. This consists of a set of unique keys (or attributes) that characterize cloud resources. A schema is also provided that defines the range of possible values for particular keys. This type of notation is commonly used amongst providers that offer CLIs. For example, the command for creating VMs in AWS CLI [AWS 2013b] expects DevOps to provide values for keys such as "image-id" and "instance-type" in order to describe the VMs to be created (refer to Code 1).

(2) Semi-structured. Semi-structured data formats, such as YAML (YAML Ain't Markup Language), XML and JSON (JavaScript Object Notation), offer a structuring mechanisms for organization of key-value pairs. They define markers to separate and enforce hierarchies among different key-value pairs. Compared to other notations, semi-structured notations are better suited for representing complex cloud resource configurations. For example, DotCloud follows YAML based resource descriptions, which include both basic and composite configuration attributes [dotCloud 2015]. Each branch in the root level represents a basic cloud resource configuration (e.g., Java VM, node.js engine, PHP engine).

(3) Domain-specific. Docker [Turnbull 2014] allows a domain-specific notation known as Dockerfiles to be written. Each specifies the configuration parameters of a particular cloud resource (refer to Code 3). DevOps may also describe a composition of a set of cloud resources using a file named docker-compose.yml (refer to Code 4).

Code 3: Dockerfile of a Python Web application

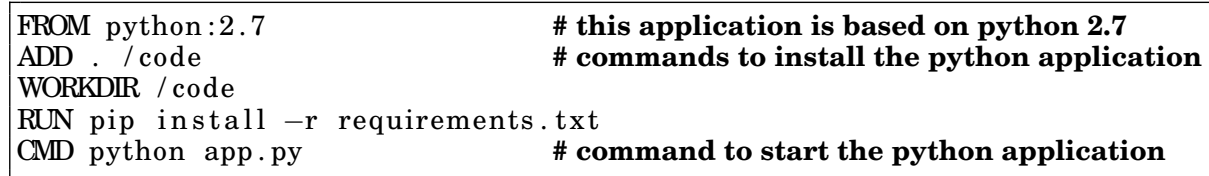

Code 4: docker-compose.yml of Web application and Redis database

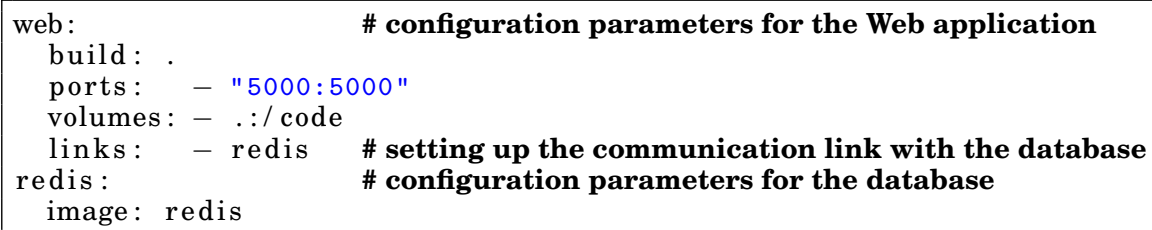

4.4.2. Visual notations. Visual programming languages abstract technical details with "visual symbols" and "graphical notations" [Chignell et al. 2010]. For example, CA AppLogic Cloud Platform [AppLogic 2015] provides a notation, with a catalog of constructs that represents elementary platform resources (e.g., databases, routers), and other visual constructs to describe composite platform resources (e.g., Web applications). Fig. 9 depicts a Web application, composed with a HTTP Gateway (i.e., IN), Web application Server (i.e., WEB5) and a network-attached storage (i.e., NAS) [Technologies 2013].

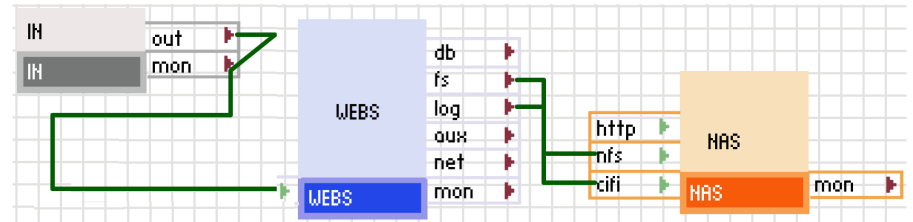

Fig. 9: Visual notation in CA-Applogic for a Web application 


\section{RESOURCE ORCHESTRATION CAPABILITIES}

Implementing orchestration processes can vary from a simple sequence of primitive actions to complex processes.

\subsection{Primitive Actions}

As per the Resource Entity Model presented in Section 4.2, Fig. 11 depicts a composite cloud resource for a Web-app runtime. This includes, an Apache-Tomcat application engine cluster with Web-apps deployed at each node. Nginx is a reverse proxy, to distribute incoming traffic to Web-apps at each node. Nagios is a monitoring service to observe the throughput of node clusters. MySQL database server persists data. MemCache is configured as a caching service, which improves the performance of database calls. For organizing primitive actions into different categories, we refer to the typical cloud resource lifecycle model (see Section 2.1). These primitive action categories are depicted as state transitions in the state chart at Fig. 10, and explained below.

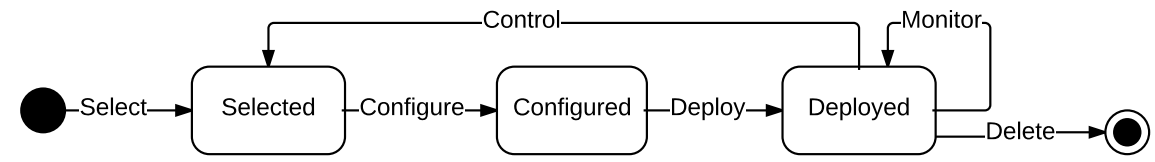

Fig. 10: State transitions of the cloud resource life cycle

(1) Select. DevOps first need to select resources that satisfy their requirements. For instance if a database is required, a viable set of database providers are evaluated and selected based on both functional (e.g., storage capacity and type of the database) and non-functional requirements (e.g., availability and cost per unit). For example, Bitnami [Bitnami 2015] provides a selection service where consumers search and select cloud resources based on intended task category (e.g., project management, Web application) and target deployment environment (e.g., personal desktops, VMWare vShpere private cloud, AWS public cloud).

(2) Configure. Next, resources are configured by defining the expected properties and relationships. For example, in AWS OpsWorks [Rosner 2013], consumers choose required resources (e.g., MySQL DB server) and provide configuration attributes (e.g., DB server with 5GB of capacity and running on port 3306) to define an expected runtime behavior. These descriptions are then submitted to the Resource Provisioning Layer (refer to Section 2.2) to create the desired cloud resources.

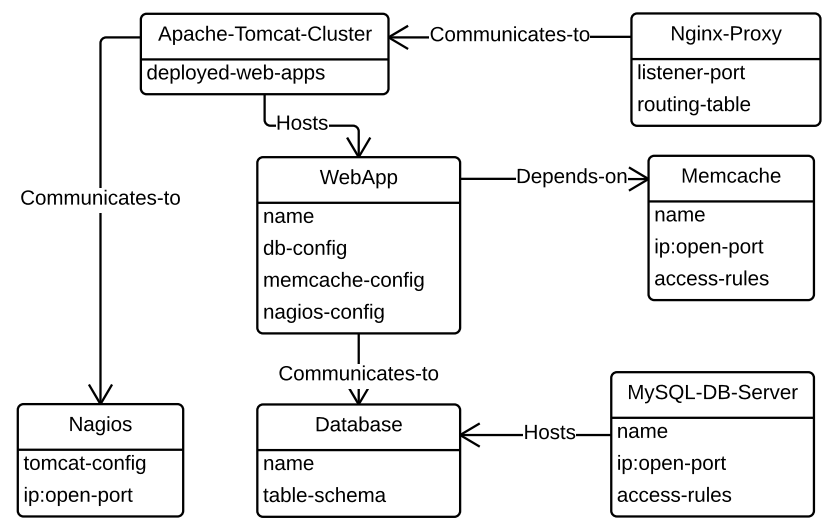

Fig. 11: A Composite Resource Infrastructure for a Web application 
(3) Deploy. Deployment involves interpreting descriptions and preparing resources into an operation and consumption-ready state. For example, administrators may use AWS-RDS API [Amazon Web Services 2015b] to provision a MySQL DBserver where the database in Fig. 11 is created. System administrators may then configure its tables manually or via an ad-hoc script (refer to Code 5).

Code 5: Linux shell commands to deploy a database server in AWS-RDS

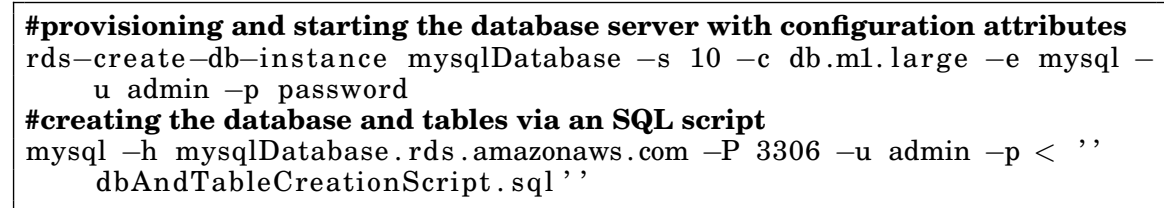

Once the component resources are constructed, relationships must be created. For example, the necessary ports and access rules should be setup within the Apache Tomcat application engine cluster and MySQL DB-server, such that requests and responses can be sent and received between the application and DB (see Fig. 11).

(4) Monitor. Once the cloud resources are operational, DevOps must monitor to ensure resources are continuously operating according to requirements. For example, a Tomcat application engine configured to be operational on $24 \times 7$, should be monitored to check that it responds to incoming requests continuously. If found otherwise, it implies the service level agreement has been violated between the provider and consumer. Nagios is a monitoring engine [Barth 2008] to specify events to be monitored (via a command definition) and receive notifications (e.g., via email). For example, Code 6 checks whether the Tomcat application engine is running or not, and a notifications may be sent (as defined in Contact definition), which sends an email to admin@abc.com when the engine is not running.

Code 6: Nagios syntax to monitor a Tomcat application engine

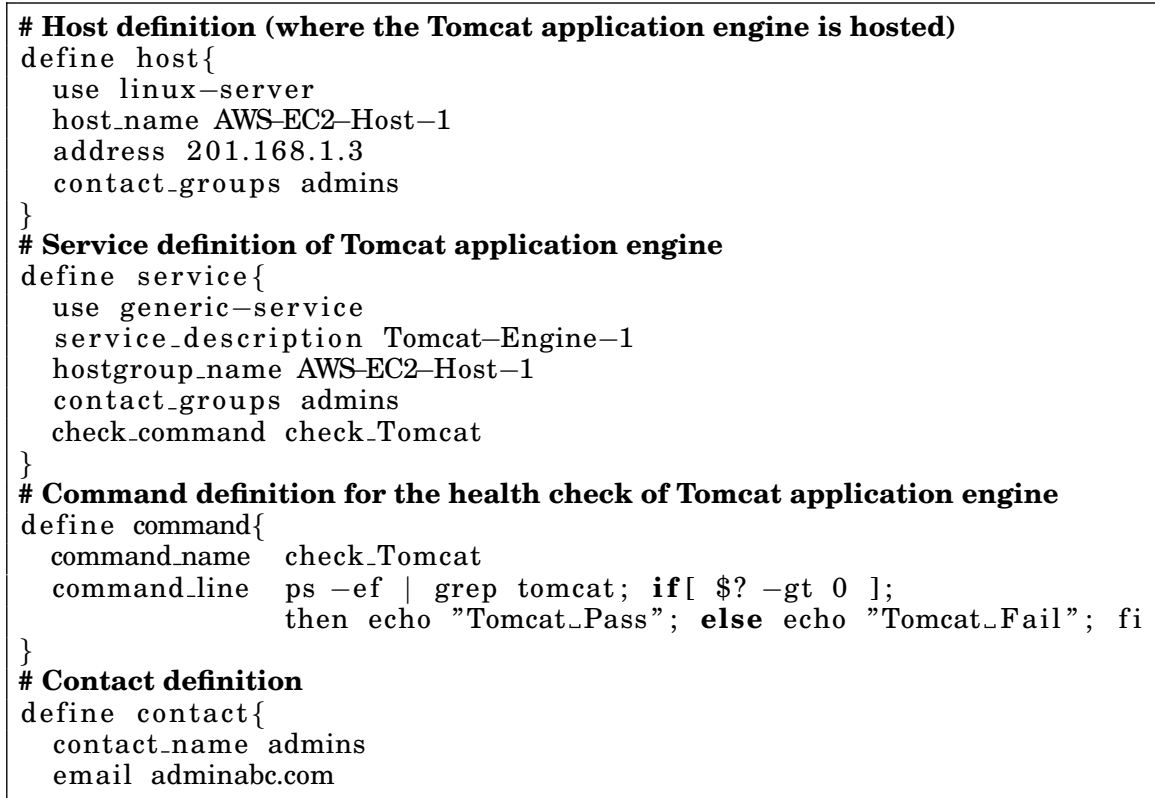


(5) Control. When cloud resources are monitored and found to be not operating according to the configured attributes, DevOps or automated processes may take necessary control actions to recover from the situation. For example, Fig. 12 depicts the orchestration logic that scales an Apache Tomcat application engine cluster when the network throughput becomes less than $95 \%$.

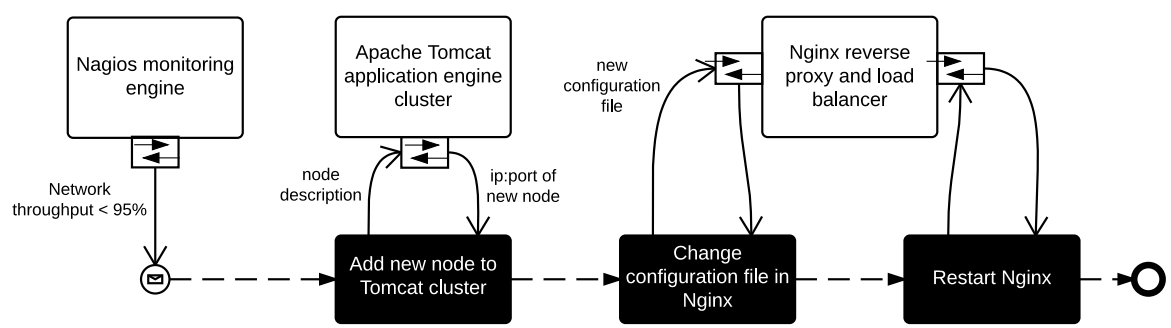

Fig. 12: Orchestration Workflow (in black and bold) for Scaling-up an Apache Tomcat Application Engine Cluster

\subsection{Orchestration Strategies}

We classify cloud resource orchestration techniques in accordance with their level of sophistication. Less sophisticated techniques require more human interventions (and vice versa), particularly to orchestrate resources in response to dynamic changes.

5.2.1. User-defined Orchestration Strategies. User-defined orchestration strategies are the most basic form of implementing cloud processes. DevOps implement orchestration processes as ad-hoc scripts, which exploit only a set of primitive actions supported by a particular orchestration language. Existing cloud resource orchestration techniques typically rely on User-defined orchestration strategies, written in general-purpose or domain-specific scripting languages [Ranjan et al. 2015; Liu et al. 2011a; Lu et al. 2013; Zeng et al. 2004]. For example, Chef Recipes [Chef 2015] follow a domain-specific scripting language that extends Ruby, to specify orchestration actions such as configuration, deployment and deletion of resources (refer to Code 7).

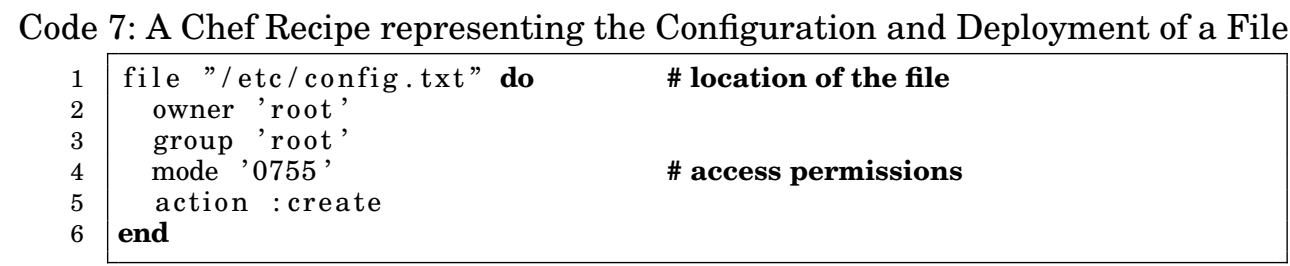

However, scaling-up or down cloud resources in dynamic environments via Userdefined orchestration processes leads to a costly and inflexible solution. This adds significant complexity, insists on extensive programming effort, calls for multiple and continuous patches, and perpetuates closed-cloud solutions. To alleviate this somewhat, tools such as Juju GUI, OpenTOSCA and VisualOps provide visual abstractions to describe deployment workflows and resource topologies [Ubuntu 2013; Binz and et al. 2013; VisualOps 2015]. For example, AWS Management Console, VisualOps, CA AppLogic and other cloud resource management tools provide control features such as restarting, scaling and migration [VisualOps 2015]. Moreover, monitoring tools such as Nagios and CloudFielder can allow DevOps to define Service Level Agreement (SLA), detect anomalies and notify about SLA violations. 
5.2.2. Rule-based Orchestration Strategies. Some providers define a reactive rule-based languages in addition to primitive actions. This means, Event-Condition-Action (ECA) rules may be specified based on pre-defined events or patterns [Michelson 2006]. When events are detected, the specified actions are auto-triggered by the orchestration engine. For example, AWS OpsWorks [Rosner 2013] supports five event types (i.e., setup, install, deploy, undeploy and shutdown). Actions consist of Chef recipes [Chef 2015]. Several research initiatives adopts this strategy: [Chapman et al. 2012; Zhang et al. 2011; Zabolotnyi et al. 2015]. Code 8 shows an elasticity rule to dynamically deploy new VMs as the "number of jobs awaiting execution increases" [Chapman et al. 2012].

Code 8: Elasticity Rule to Scale VMs (adapted from Chapman et al. 2012)

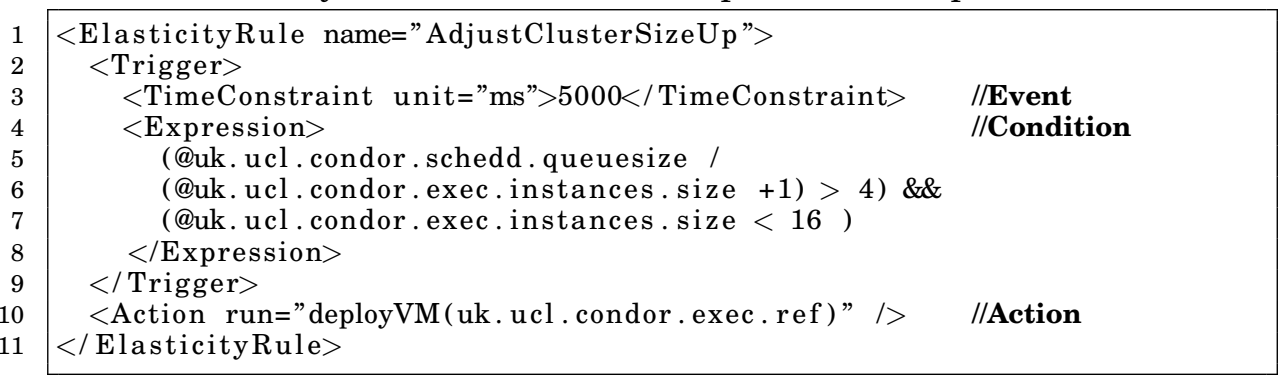

5.2.3. Autonomic Orchestration Strategies. With the expanding complexity of cloud-based systems, orchestration tasks become too cumbersome to be carried-out largely with human-assisted techniques including user-defined and rule-based strategies. Autonomic orchestration strategies, the highest level of sophistication, refer to selfmanaging features of cloud resources [Toosi et al. 2014; Singh and Chana 2015]. For instance, endowing resources with self-management capabilities has the potential to enhance high availability of cloud resources, e.g., through dynamic (re-)configuration, in order to maintain the expected quality of service in the presence of faults, variable environmental conditions, and changes in user requirements [Singh and Chana 2015; Zhan et al. 2015a; Cheng and Garlan 2012; Yuan et al. 2014]. For example, an autonomic orchestration process automatically scales up or down running applications by analyzing the recent resource consumption statistics. This implies, orchestration techniques intelligently make certain decisions when managing cloud resources without taking any instructions from users [Parashar and Hariri 2005].

It should be noted that, in terms of self-managing cloud resources services, technology is still in the early stages. CometCloud is an example of efforts in this direction [Kim and Parashar 2011]. Supported features include budget-, deadline-, and workload-based deployment of cloud-based applications. Other efforts include automatic re-configuration of resources to meet evolving application resource requirements and QoS [Zhan et al. 2015a; Singh and Chana 2015; Toosi et al. 2014]. For example, Fuzzy BPM-aware Auto-Scaler scales-up or down VMs based on Key Performance Indicators (KPIs) of the VMs and the deployed business processes within the VMs [Schulte et al. 2015; Mamdani 1974]. Other research initiatives trigger orchestration actions and processes based on analysing user requirements (e.g., SLAs); end-user context (e.g., geolocations and device configurations); and environmental properties (e.g., unit cost per resource, processing speed of VMs) [Wei and Blake 2013; Gravier et al. 2015; Menzel et al. 2015; Zhang et al. 2012a; Zabolotnyi et al. 2014; Fang et al. 2012].

Learning-based methods, based on historical or simulated data, have been applied to support autonomic cloud resources orchestration. Xu et al., Sadeka et al., ASAP and ORMDSS propose neural-network models that are trained using different workload 
scenarios, and used to determine an optimal or near-optimal configuration of VMs and software appliances [Xu et al. 2012; Islam et al. 2012; Jiang et al. 2011; Ramezani et al. 2013]. Antonescu et al. propose a learning-based technique to migrate and provision cloud-based mobile services based on the mobility of users [Antonescu et al. 2013].

Various heuristic-based resource allocation and migration algorithms have also been proposed to support autonomic orchestration of cloud resources [Mishra et al. 2012; Pandey et al. 2010; Beloglazov et al. 2012; Iqbal et al. 2011]. Some of these are based on pre-defined policies that determine which type of VMs should be provisioned to which data centers; while optimizing the energy consumption [Beloglazov et al. 2012].

We identify several other methods, which include formally defined abstractions for specifying automated resource orchestration. Namely, Closures, Promises and Aspects.

Closures encapsulate orchestration commands as black boxes; this aids to reduce management complexity and costs [Couch et al. 2003; Burgess and Couch 2006]. Its behavior can be thought of as the sum of its transactions with the outside world, such that each output from a closure is a function of all input received. Inputs take the form of events and streams. Closures are adopted in CFEngine [Burgess and College 1995].

Promises model the way cloud resources commit to certain behaviors [Burgess and Couch 2006; Bergstra and Burgess 2014]. It allows cloud resources to become more autonomous and self-sufficient in dynamic environments. In CFEngine, Promises are implemented as policies that modify resources, such as those in non-conforming states which are transformed into conforming states [Burgess and College 1995]. Effectively, this approach immunizes cloud resources against potential deterioration by continuously repairing those non-conforming. Promises are also idempotent; they will do nothing unless non-conformity is discovered. This technique have been applied to verification and knowledge management of cloud orchestration [Burgess 2011; Burgess 2009].

Aspects are an abstraction for organizing Promises into distributed bundles and constellation [Burgess 2007]. Aspects are introduced over Promises to describe complex orchestrations which need to be dealt with by multiple Promises simultaneously.

\subsection{Language Paradigm}

Language paradigm is an "approach of programming based on a coherent set of principles and practices", which determine its "suitability for solving certain types of problems" [Van Roy et al. 2009]. We have identified the following language paradigms used in cloud orchestration systems (e.g., Puppet, Chef, Juju, Docker, SmartFrog, AWS OpsWorks), and research efforts [Cui et al. 2013; Chieu and at al. 2010; Goldsack and at al. 2009; Delaet et al. 2010; Konstantinou and et al. 2009; Wilson 2009]:

5.3.1. Imperative Programming. We have identified three sub-categories:

- Script-based. DevOps widely adopt scripting languages (e.g., JavaScript, Python, Bash) to implement cloud resource orchestration processes. Providers that use this method include Docker and Vagrant [Turnbull 2014; Hashimoto 2013].

- Flow-based Programming. The primitive constructs of flow-based orchestration languages are data-flow and control-flow connectors. This approach is based on on service composition and business process modeling languages (e.g., BPEL, BPMN [OMG 2011; Juric and Weerasiri 2014]. BPMN4TOSCA [Kopp and et al. 2012] (which include four BPMN extensions) and CloudBase [Weerasiri et al. 2015a] extend BPMN to implement orchestration processes of cloud applications.

- Rule-based Programming. ECA rules are specified by associating a sequence of configuration, deployment or re-configuration actions for each of possible events [Ubuntu 2013]. Code 8 exemplifies an ECA rule that dynamically deploys new VMs as the "number of jobs awaiting execution increases" [Chapman et al. 2012]. 
5.3.2. Declarative Programming. We have identified three sub-categories:

- Markup Languages. Plush is a tool to deploy, monitor and control distributed software applications. It advocates an XML-based language to model and deploy software components [Albrecht and et al. 2011].

- Query-based. Query-based orchestration languages model cloud resources as structured data (e.g., tables, graphs, trees) and provide actions (e.g., create, read, update and delete) for processing structured data. [Liu et al. 2011b; Liu et al. 2011a] represents cloud resources as a tree-like data structure and provide declarative primitives to create, delete and update cloud resources.

- Constraint Programming. Constraint programming enables automatic generation of cloud resource configurations from declarative constraint specifications [Danninger 2015; Sawyer et al. 2012]. For example, CFEngine automatically determines the steps required to create and update resource configurations by analyzing constraint specifications and recent changes within the operating environment [Burgess and College 1995].

\section{USER TYPES}

We identify three types of users involved in orchestrating cloud resources, these are namely DevOps, Application Developers, and Domain-Experts. DevOps is an emerging role to consolidate application developers and system administrators. To effectively manage resources, complex orchestration processes needs to be carried out, (e.g., setting up an application testing environment, testing application updates, migrating the tested environment to the production, scaling the production environment based on usage patterns). DevOps are responsible for optimizing and automating those orchestration processes, which improves the quality of software development and continuous delivery processes. The traditional application developers role is also important including the use of cloud for resources management. However, not all application developers are DevOps (which implies the distinction of roles in our analysis). For example, developers may just be responsible to write Java code that are later deployed on Heroku.

A domain expert is specialized in a specific domain (e.g., biologists, teachers), and may use cloud resources for their work processes. For example, a Harvard University lecturer for Introduction to Computer Science Course [Malan 2015] may create a virtual machine named CS50 Appliance 19 [CS50 2015], which includes all software required by students to develop, test, deploy and execute code. Domain experts have very little or no programming expertise. For this reason, it is imperative that domain experts are provided with end-user-oriented or domain-specific orchestration languages. RightScale Self-Service allows domain-experts to automatically provision and orchestrate Software resources based on high-level policies [RightScale 2016]. It also provides a Web-based portal, whereby domain-experts can deploy resources by specifying non-technical resource attributes and assess their financial costs.

\section{RUNTIME ENVIRONMENT}

The runtime environment for cloud orchestration relies on three orthogonal concerns: (a) virtualization technique; (b) execution model; and (c) target environment.

\subsection{Virtualization Technique}

Virtualization is the key technique that transforms cloud resource descriptions into concrete resources; it provisions hardware and software constituents without upfront capital expenditure [Chapman et al. 2012]. It addresses three main concerns: (a) performance isolation; (b) data isolation; and (c) execution isolation [Gupta et al. 2006]. Data interference is the unintended data sharing (e.g., file systems) across different 
resources. Execution interference is the effect on the runtime state (e.g., failures) of one resource to another resource. Performance interference is the influence of the performance of one resource to another, where both share the same underlying resources.

We discuss two types of virtualization techniques that are commonly adopted by cloud resource orchestration techniques:

- OS-level Hypervisor. The virtualization component runs on top of a host operating system, with VMs installed with a guest operating system (Fig. 13 (left)). The virtualization component accesses a shared pool of resources (e.g., memory, CPU and system calls) through the host operating system and partitions resources across operating systems. For example, AWS EC2 service uses an extended version of Xen as their OS-level hypervisor to provision EC2 virtual machines.

- Environment-level Container Manager. The virtualization component runs on top of the kernel of a host operating system, similar to OS-level hypervisors. In contrast however, the hardware layer is not virtualized but use features of the operating system kernel to create lightweight virtualized operating system environments, i.e. containers, (Fig. 13 (right)). For example, LXCs (Linux containers) are built by leveraging cgroups and namespace features of the Linux kernel [LinuxContainers.org 2015; Rosen 2013]. Environment-level containers do not require installing separate guest operating systems on each container - they share the hardware layer and host operating system kernel layer across all containers. This is a resource isolation mechanism with little overhead compared to OS-level hypervisors. Docker is a container manager on top of the Linux OS [Turnbull 2014].

- Minimum-OS Unikernels. The virtualized component implements the bare minimum operating system's kernel libraries; just enough to support successful execution of the component. Unlike containers, Unikernels-based (e.g., MirageOS, Rump Kernel, Clive) virtualized components [Oliver 2015] do not share OS kernel libraries with other containerized instances. By presenting the ability to compose application components that are not only lightweight while having the security features of OS-Level Hypervisors, Unikernels have starting to emerge as a viable alternative to overcome the current security concerns relevant to Container-based (hypervisor-free) approaches. Moreover, application developers have explicit control over core security areas, as they can choose which kernel library to package or turn off by default. Similar to containers, Unikernels are much lightweight [Oliver 2015; Darvell 2016] as compared to traditional, full-blown Virtual Machines (VMs) that are hosted on top of OS-level hypervisors (e.g., Hyper-V, Xen).
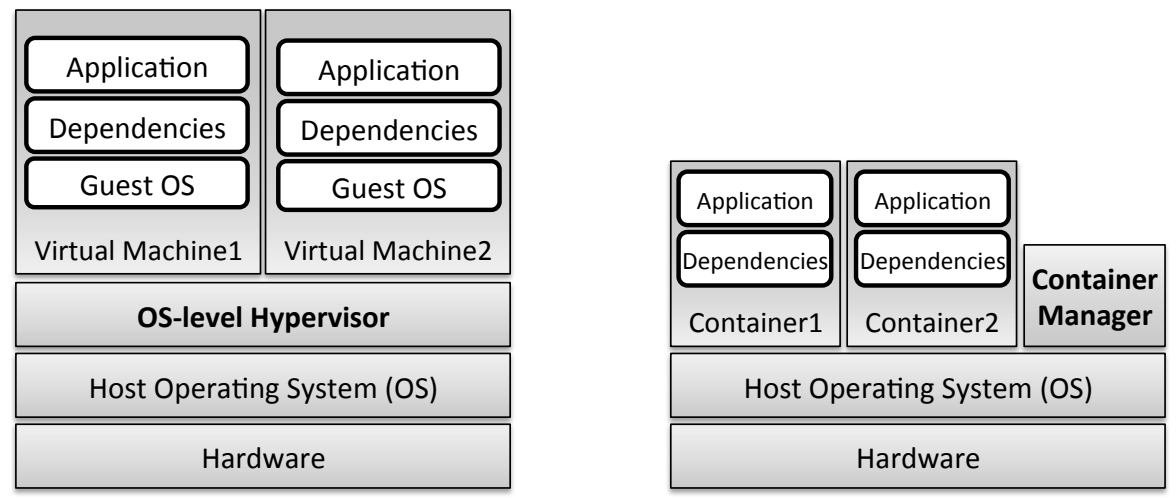

Fig. 13: OS-level hypervisor (left) vs. Container Manager (right) 


\subsection{Execution Model}

The execution model refers to how a particular orchestration process distributes and performs tasks. We identify two main types of execution models:

- Centralized Orchestration. In this model, the execution manager performs all the tasks of an orchestration process. If tasks are dispersed across a set of machines within a distributed environment, the centralized manager directly issues commands to perform the orchestration. For example, VMWare vSphere [Lowe 2011] is a virtual machine management tool that creates and manages VMs on top of a single host machine. Ansible performs as a central manager, which directly issues orchestration commands via the SSH (Secure Shell) protocol; such that the issued commands are received by remote machines [Mohaan and Raithatha 2014].

- De-Centralized Orchestration. In this model, all participating machines are required to install an agent supplied by the orchestration provider. During execution, tasks are delegated to the agent - which is thereby responsible to perform the actual orchestration tasks. Agents are only aware of their delegated tasks, and not about tasks assigned to other agents. For example, Puppet supports agentbased orchestration in which there is a central server that stores orchestration processes. Agent machines periodically poll the central server for orchestration tasks and perform those tasks. Puppet follows a model based on Promises (refer to Section 5.2.3) to avoid potential inconsistencies in autonomous and de-centralized orchestrations [Bergstra and Burgess 2008]. Kirshnick et. al. propose a peer-topeer architecture, a highly scalable and fault-tolerant architecture with no central orchestration server, to automatically deploy software components across a pool of virtual machines [Kirschnick and et al. 2012].

\subsection{Target Environment}

7.3.1. Public Cloud. Public cloud providers, such as AWS provides a range of orchestration techniques (e.g., AWS Command Line Interface, AWS CloudFormation, AWS OpsWorks), each of which suits different types of users (e.g., system administrators, DevOps, developers) to configure, deploy and control cloud resources. Alternatively, there are third-party cloud resource orchestration techniques that provide plug-ins to integrate with public cloud providers. For example VisualOps provides a graphical interface to configure and visualize VMs deployed across different regions (e.g., Europe, Australiasia) in the AWS environment [VisualOps 2015].

7.3.2. Private Cloud. Private cloud resource providers, such as VMWare and OpenStack offer VMWare vSphere and Heat respectively to configure and manage virtual machines within a private network [Lowe 2011; OpenStack.org 2015b]. Additionally, third-party tooling such as Juju, Ansible, Chef and Puppet [Ubuntu 2013; Mohaan and Raithatha 2014; Sabharwal 2014; Labs 2015a; Kanies 2006] support resource configuration, deployment and control in OpenStack-based private cloud deployments.

7.3.3. Federated Cloud. Tools for orchestrating federated cloud resources have been introduced in both research and industry [Toosi et al. 2014; Hajjat et al. 2011]. They either: (a) define a unified cloud resource orchestration language which must be conformed to by all participating providers [Weerasiri et al. 2015a; Wettinger and et al. 2014]; or (b) provide a pluggable architecture that interprets different orchestration languages of participating providers [Wettinger et al. 2014; Weerasiri et al. 2015b].

For example, TOSCA is an open standard for representing and orchestrating cloud resources [OASIS 2013; Binz and et al. 2013]. It describes a federated cloud resource using a Service Template. This template captures the topology of component resources, and sets a plan for orchestrating those resources. 
Techniques for capturing a unified representation, as well as enabling orchestration of cloud resources amongst diverse providers have been studied by research [Moscato and et al. 2011; Smit et al. 2013] and implemented as language libraries [Foundation 2014c; Tidwell 2009; Foundation 2014a; Foundation 2015c]. On the other hand, Ansible provides a suite of distinct language modules each of which publishes an orchestration interface for a specific resource type offered by a particular resource provider (e.g., AWS, Rackspace, Azure, VMWare) [Ansible 2015]. Ansible is thus able to implement scripts by reusing a set of modules to model and orchestrate federated cloud resources.

Federated Cloud is a key factor to facilitate switching providers, i.e. avoiding vendor lock-in and optimizing cost-to-performance tradeoffs. Recognizing this competitive edge, cloud resource orchestration tools and techniques are attempting to expand their capability to compose, deploy and manage applications across multiple cloud providers. Nonetheless, the federated cloud model is nontrivial to design, it is also difficult to implement generic resource orchestrators that can work with various providers. We (and others [Toosi et al. 2014]) believe that Federated Cloud can be realized either using Multi-Cloud or Hybrid Cloud abstractions. When considering the Federated Cloud environment, an organization must consider security and regulatory compliance requirements as they tend to vary considerably across providers.

Resource orchestration in Multi-Cloud environments refer to transparently integrating IaaS and PaaS resources offered by multiple public cloud providers as part of single application composition. With a Multi-Cloud orchestrator, administrators can manage and automate both application movement across public clouds and the communication among resources hosted in different clouds. Resource orchestrators provided by RightScale, EngineYard, and CohesiveFT support application deployment across multiple clouds (e.g., Amazon Web Services, Microsoft Azure, HP Cloud) by implementing adapter layer (for example based on APIs such as Apache jclouds) that hides the low level technical complexity (e.g., hypervisor type, authentication, authorization, networking) of heterogeneous, multiple clouds from high-level application composition (configuration, monitoring, run-time adaptation).

On the other hand, Resource Orchestration in Hybrid Cloud environments refer to transparently shedding excess application workload to one or more external public clouds in the event private cloud resources are not able to cope with the demand for computing capacity spikes. The major advantage of Hybrid Cloud environment is that an organization only pays for extra resource capacity only when they are needed. Resource orchestrators provided by commercial PaaS providers such as CloudSwitch (supports bursting of in-house workload to AWS and has been recently acquired by Verizon) and EU FP7 Projects such as OPTIMIS and SeaClouds support deployment of applications in Hybrid Cloud environments.

\section{KNOWLEDGE REUSE}

Knowledge reuse frameworks are based on four main pillars: (a) Knowledge Representation; (b) Knowledge Acquisition; (c) Knowledge Curation; and (d) Knowledge Discovery. Knowledge representation techniques are presented in Section 8.1. We then discuss various methods used for knowledge acquisition, curation and discovery, in Section 8.2.

\subsection{Reused Artifact}

An artifact may be atomic (e.g., a resource description or orchestration rule); or composite including multiple interrelated elements (e.g, deployment workflow). Reuse artifacts can be distinguished as template or concrete. Concrete artifacts are fullydeveloped orchestration solutions. Template artifacts are generalized solutions, which need manual adaptations (e.g., initializing configuration parameters) before reuse. Considering the above, we have identified the following variety of reuse artifacts: 
8.1.1. Resource Description Templates. Most enterprise-ready cloud orchestration providers support both concrete and template resource description repositories for knowledge-reuse. For example, Google Container Engine, Docker and Juju offer cloud knowledge repositories (i.e, Google Container Registry, Docker Hub and Juju Charm Store)[Google 2015a; Docker 2015a; Canonical 2015; Services 2015b]. Docker Hub enables sharing and reusing resource descriptions by means of Docker Images; which represent resource deployment descriptions (e.g., mongoDB database, nginx reverse proxy server) with the required dependencies. Docker Hub may be used to discover, configure and deploy existing Images. Template Images are associated with a set of configuration parameters (e.g., access credentials of a database server Image), which are initialized by users before the deployment; while concrete Images have pre-initialized configuration parameters.

8.1.2. Resource Snapshots. A snapshot of a cloud resource includes not just its description but also a specific runtime state (e.g., deployed and started application server). In contrast to reusing resource description templates, snapshots additionally embed information about the execution of the orchestration process. For example, Snaps in terminal.com and VMware Snapshots provide resource snapshots [Inc. 2015; VMware 2015]. Users of terminal.com (e.g., application developers) may specify, deploy and share Snaps with other users (e.g., QA engineers, system administrators) - who may test, monitor and control those Snaps.

8.1.3. Miscellaneous. DevOps create and publish DockerFiles, which are textual resource descriptions of Docker Images; they may then be shared on code repositories such as GitHub. Instructions for how to configure and deploy the specific DockerFile into a Docker Container may also be shared. Albeit these instructions can only be interpreted by humans (not machine read).

\subsection{Reuse Techniques}

Given an artifact for reuse, it is imperative to identify different techniques that can be applied in practice to enable its reuse. We identify the following three categories:

8.2.1. Search Indexes. Ansible, Puppet and Chef provide search indexes based on resource description attributes (e.g, artifact name, owner, version and created date) [Mohaan and Raithatha 2014; Labs 2015a; Sabharwal 2014]. This assumes that users know the exact (or nearly exact) attributes values in order to query for potential artifacts to reuse. There are more advanced search indexes (e.g., Bitnami) which accept query inputs such as intended task category (e.g., project management) and target deployment environment (e.g., AWS EC2 public cloud, VMWare vSphere private cloud).

8.2.2. Recommendation. This approach implies proactively suggesting a set of potential artifacts to facilitate the orchestration. Compared to search indexes, recommended artifacts are suggested based on application profiles, usage histories, contexts, etc [Resnick and Varian 1997; Weerasiri and Benatallah 2015; Zhang et al. 2012c]. For example, AWS marketplace suggests virtual appliances based on users' ratings and comments. Additionally when users choose a particular virtual appliance (e.g., http server), a list of related virtual appliances (e.g., http load balancer) is recommended that can be deployed along with the chosen appliance.

8.2.3. Community-driven Techniques. Leveraging user-expertise to facilitate knowledgereuse is a popular choice amongst many enterprise-level cloud providers.

- Resource Repositories. As earlier mentioned, online databases such as Docker Hub and AWS EC2 Container Registry act as Git-like version-control repositories [Docker 2015a; Services 2015b]. Some communities like Bitnami [Bitnami 2015] 
restrict all but authorized developers to register resource artifacts. Other such as, Ubuntu Juju [Juju 2015a] and Puppet [Labs 2015b] implement strict curation policies (e.g., licensing, naming conventions, idempotency of orchestration rules) when sharing artifacts. Yet while other communities such as Docker Hub [Docker 2015a] do not enforce curation policies, albeit implement reputation schemes to collectively estimate quality and correctness of resource artifacts.

- Forums, Blogs and Wikis. Forums allow users to post questions and ideas, and receive targeted answers and comments from other users. For example, Puppet provides a forum for DevOps to post, query, answer and rate questions. Blogs usually contain information authored by a single user or organization. For example, Chef community posts blog articles about artifact development best practices, updates to the orchestration language and other related news. Wikis are communitydriven collaborative environments that are particularly useful for small teams to maintain documentation of cloud resources. For example, DevOps can keep track of the list of deployed VMs. DevOp may then update the wiki whenever they make any changes (e.g., installing software, operating system updates).

\section{APPLYING THE TAXONOMY: EVALUATION OF CLOUD RESOURCE ORCHESTRATION TECHNIQUES}

In consolidation of the foregoing discussion, we organize the analysis of state of the art by characterizing techniques and tools along the main dimensions of our taxonomy (as presented in Section 3). We include well-known enterprise tools and frameworks, as well as initiatives derived from a wide selection of research literature.

\subsection{Selection Process}

Careful consideration was applied in the selection of relevant tools for our analysis; this entailed several phases of investigation: Initially, 20 orchestration tools were chosen out of a set heavily advocated by the DevOps community. We experimented with those tools to understand the main dimensions that are common among to these tools Based on our observations, we were able to derive the initial draft of our taxonomy. Furthermore, we derived analysis tables that summarize how each tool accaording to the identified dimensions of the initial taxonomy. We then chose a selection of research initiatives from leading, critically-reviewed research proceedings (research and demonstration tracks), magazines, and journals articles that were relevant to the domain from the year 2004 onwards. In particular, these included the following conferences: Cloud Computing (CLOUD), Cloud Engineering (IC2E), Service-Oriented Computing (ICSOC), Advanced Information Systems Engineering (CAiSE), Large Installation System Administration (LISA), Database Systems for Advanced Applications (DASFAA), Cooperative Information Systems (CoopIS), Cloud Computing and Services Science (CLOSER), Utility and Cloud Computing (UCC). And the following journals: ACM Computing Surveys (CSUR), ACM Transactions on Internet Technology (TOIT), IEEE Internet Computing, IEEE Transactions on Network and Service Management (TNSM), IEEE Transactions of Cloud Computing (TCC), Journal of Systems and Software (JSS). We analyzed these initiatives and further revised our taxonomy and comparison tables based on our findings.

Ultimately, 11 different cloud orchestration approaches were selected for analysis, namely: AWS OpsWorks [Rosner 2013], AWS CloudFormation [Amazon 2011], VMWare vSphere [Lowe 2011], Heroku [Middleton et al. 2013], Puppet [Kanies 2006; Labs 2015a], Juju [Ubuntu 2013], Docker [Turnbull 2014], OpenTOSCA [Binz and et al. 2013], CFEngine [Burgess and College 1995], Plush [Albrecht and et al. 2011], and SmartFrog [Goldsack et al. 2009]. 


\subsection{Resources and User Types}

Table I maps the selected orchestration techniques onto the Resources and User Types dimensions described in Section 4 and 6. The supported resource types, access methods and representation notations immensely influence the type of users. Therefore to appreciate this correlation, we present our analysis of these two dimensions together.

Accordingly, by studying the characteristics relative to these two dimensions, we summarize our findings as follows:

- 8 out of 11 approaches utilize Domain-specific representation notations - however there are an assortment of other notations with the same or similar representation capabilities (as cited in Section 4.4). This underlines the factual assertion and suitability of domain-specific notations in the field of cloud resource orchestration.

- 10 out of 11 approaches support CLI-based resources access; from which 7 also provide API-based access. Due to the fact that Linux and Unix based systems are managed via CLIs, the current DevOps community is heavily equipped with CLIbased system administration skills. To manage applications across public and private clouds, providing APIs and SDKs for programmatically accessing resources become an important requirement.

- 10 out of 11 approaches support representing platform resources. In general, cloud resource orchestration vastly remains the prerogative of professional DevOps, although the adoption of end-user intuitive visual abstractions is emerging.

As future directions, it will be vital to provide effective end-user oriented representation capabilities. This will be complimented by diversifying techniques for resource representations. More specifically:

- End-User empowered declarative representation. We identify end-users as an important and emerging category of user-type for orchestration techniques in future. Accordingly, end-users should be able to easily and declaratively represent cloud resources. As well as, access, configure, compose, and analyze simple yet powerful composite cloud resources. Currently, even sophisticated DevOps are often forced to resort to grasping different low-level resource access methods, and procedural language paradigms, to create and manage complex cloud resources.

- Adoption of open-standards. Furthermore, while it is still not prevalent, the adoption of open standards (e.g., TOSCA, OVF, OCF) to represent reuse artifacts [OASIS 2013; Crosby and et al. 2009; Foundation 2015b] would significantly assist DevOps to build portable and interoperable configurations across different cloud providers. Accordingly, we believe any type of representation paradigm would benefit by adopting open standards.

Table I: The Resource and User Type Dimensions of the Selected Platforms

\begin{tabular}{|c|c|c|c|c|c|c|c|}
\hline & \multicolumn{6}{|c|}{ Resource } & \multirow[t]{3}{*}{ User Type } \\
\hline & \multirow[t]{2}{*}{ Res. Types } & \multirow{2}{*}{$\begin{array}{l}\text { Res. } \\
\text { Represen- } \\
\text { tation } \\
\text { Notation }\end{array}$} & \multicolumn{3}{|c|}{ Res. Entity Model } & \multirow{2}{*}{$\begin{array}{l}\text { Res. Access } \\
\text { Method }\end{array}$} & \\
\hline & & & Entities & Relationships & Constraints & & \\
\hline $\begin{array}{l}\text { AWS } \\
\text { OpsWorks }\end{array}$ & $\begin{array}{l}\text { Platform } \\
\text { Resources }\end{array}$ & $\begin{array}{l}\text { Domain- } \\
\text { specific } \\
\text { notation } \\
\text { based } \\
\text { on Chef } \\
\text { cookbooks }\end{array}$ & $\begin{array}{l}\text { Support defining } \\
\text { composite trees } \\
\text { from component } \\
\text { entities. Compo- } \\
\text { nent entities are } \\
\text { are Web applica- } \\
\text { tion components. }\end{array}$ & $\begin{array}{l}\text { Support Containment rela- } \\
\text { tionship to compose a set of } \\
\text { related resources required for } \\
\text { a Web application }\end{array}$ & $\begin{array}{l}\text { Constraints are al- } \\
\text { lowed in entities as } \\
\text { attributes and rules (eg } \\
\text { - auto-scaling rules in } \\
\text { Layer) }\end{array}$ & $\begin{array}{l}\text { Web based } \\
\text { GUI, CLI, } \\
\text { SDK, APIs }\end{array}$ & DevOps \\
\hline
\end{tabular}




\begin{tabular}{|c|c|c|c|c|c|c|c|}
\hline & \multicolumn{6}{|c|}{ Resource } & \multirow[t]{3}{*}{ User Type } \\
\hline & \multirow[t]{2}{*}{ Res. Types } & \multirow{2}{*}{$\begin{array}{l}\text { Res. } \\
\text { Represen- } \\
\text { tation } \\
\text { Notation }\end{array}$} & \multicolumn{3}{|c|}{ Res. Entity Model } & \multirow{2}{*}{$\begin{array}{l}\text { Res. Access } \\
\text { Method }\end{array}$} & \\
\hline & & & Entities & Relationships & Constraints & & \\
\hline $\begin{array}{l}\text { AWS } \\
\text { CloudFor- } \\
\text { mation }\end{array}$ & $\begin{array}{l}\text { Infrastructure } \\
\text { and Platform } \\
\text { resources }\end{array}$ & JSON & $\begin{array}{l}\text { Support defining } \\
\text { composite graphs } \\
\text { from component } \\
\text { entities }\end{array}$ & $\begin{array}{l}\text { (1) Support Dependency rela- } \\
\text { tionships between resources. } \\
\text { (2) Support Containment } \\
\text { relationships to group all the } \\
\text { related resources }\end{array}$ & $\begin{array}{l}\text { (2) Local attributes } \\
\text { in resource entities } \\
\text { (2) Attributes can be } \\
\text { defined to apply on all } \\
\text { the resource entities }\end{array}$ & CLI, APIs & DevOps \\
\hline $\begin{array}{l}\text { VMWare } \\
\text { vSphere }\end{array}$ & $\begin{array}{l}\text { Virtual Ma- } \\
\text { chines }\end{array}$ & Visual & $\begin{array}{l}\text { Top level resource } \\
\text { is a ServiceIn- } \\
\text { stance (a data } \\
\text { center). Service- } \\
\text { Instance can be } \\
\text { modeled a set of } \\
\text { VMs which can } \\
\text { be composed of } \\
\text { component enti- } \\
\text { ties like network, } \\
\text { alarm }\end{array}$ & $\begin{array}{l}\text { (1) Support Dependency rela- } \\
\text { tionships between resources. } \\
\text { (usually these relationships } \\
\text { can be modeled between the } \\
\text { component resources within } \\
\text { VMs) (2) Support Contain- } \\
\text { ment relationships to group all } \\
\text { the related VMs }\end{array}$ & $\begin{array}{l}\text { Support attributes in } \\
\text { resource entities to } \\
\text { configure VMs }\end{array}$ & $\begin{array}{l}\text { Desktop } \\
\text { based GUI, } \\
\text { CLI, APIs }\end{array}$ & $\begin{array}{l}\text { System } \\
\text { Admin- } \\
\text { istrators }\end{array}$ \\
\hline Heroku & Platform & $\begin{array}{l}\text { Domain- } \\
\text { specific }\end{array}$ & $\begin{array}{l}\text { Support defining } \\
\text { composite trees } \\
\text { from component } \\
\text { entities }\end{array}$ & $\begin{array}{l}\text { (1) Support Containment } \\
\text { relationships to group a set } \\
\text { of Dynos which belong to a } \\
\text { particular app. (2) Support } \\
\text { Dependency relationships } \\
\text { (e.g., pom.xml in java apps) }\end{array}$ & $\begin{array}{l}\text { (1)Support attribute } \\
\text { based constraints } \\
\text { in resource entities. } \\
\text { (2) Policies can be } \\
\text { specified on particular } \\
\text { entities (eg., At least } \\
\text { one Web Dyno entity } \\
\text { should exist in each } \\
\text { App entity) }\end{array}$ & CLI, APIs & DevOps \\
\hline Puppet & $\begin{array}{l}\text { Platform } \\
\text { Resources }\end{array}$ & $\begin{array}{l}\text { Domain- } \\
\text { specific }\end{array}$ & $\begin{array}{l}\text { (1) Supports a } \\
\text { graph of resource } \\
\text { entities (2) Entity } \\
\text { types include } \\
\text { files, packages } \\
\text { like resource that } \\
\text { can be composed } \\
\text { to model a ma- } \\
\text { chine (3) Top } \\
\text { level Composite } \\
\text { entity repre- } \\
\text { sent a Machine } \\
\text { (Physical/Virtual) }\end{array}$ & $\begin{array}{l}\text { (1) Support Dependency } \\
\text { relationships which results the } \\
\text { deployment behavior among } \\
\text { resource entities (2) Hosting } \\
\text { relationships to specify which } \\
\text { resource entities should be } \\
\text { deployed on which machines }\end{array}$ & $\begin{array}{l}\text { Resource entity } \\
\text { specific constraints are } \\
\text { provided as attributes. } \\
\text { Puppet also define a } \\
\text { hierarchical structure } \\
\text { to categorize resource } \\
\text { entities such that } \\
\text { constraints defined in } \\
\text { parent are inherited to } \\
\text { children }\end{array}$ & $\begin{array}{l}\text { CLI, APIs, } \\
\text { Web based } \\
\text { GUI }\end{array}$ & DevOps \\
\hline Juju & $\begin{array}{l}\text { Infrastructure } \\
\text { and Platform } \\
\text { resources }\end{array}$ & YAML & $\begin{array}{l}\text { (1) Support a } \\
\text { graph of resource } \\
\text { entities }\end{array}$ & $\begin{array}{l}\text { (2) Dependency relation- } \\
\text { ships between Charms (e.g., } \\
\text { require, provide interfaces) } \\
\text { (2) Containment relationship } \\
\text { (e.g., between Charms and } \\
\text { the Provider) (3) Hosting } \\
\text { relationship (e.g., between a } \\
\text { service-unit and a Machine/- } \\
\text { Container) }\end{array}$ & $\begin{array}{l}\text { Support entity and } \\
\text { relationship spe- } \\
\text { cific constraints via } \\
\text { attributes }\end{array}$ & $\begin{array}{l}\text { CLI, Web } \\
\text { based GUI }\end{array}$ & DevOps \\
\hline Docker & $\begin{array}{l}\text { Platform } \\
\text { Resources }\end{array}$ & $\begin{array}{l}\text { Domain- } \\
\text { specific }\end{array}$ & $\begin{array}{l}\text { Support a graph } \\
\text { of resource } \\
\text { entities }\end{array}$ & $\begin{array}{l}\text { (1) Communication rela- } \\
\text { tionships (2) Dependency } \\
\text { relationships (3) Hosting } \\
\text { relationship }\end{array}$ & $\begin{array}{l}\text { Entity specific con- } \\
\text { straints via attributes }\end{array}$ & CLI, APIs & DevOps \\
\hline $\begin{array}{l}\text { Open- } \\
\text { TOSCA }\end{array}$ & $\begin{array}{l}\text { Infrastructure } \\
\text { and Platform } \\
\text { resources }\end{array}$ & $\begin{array}{l}\text { Visual } \\
\text { notation }\end{array}$ & $\begin{array}{l}\text { Support a graph } \\
\text { of resource } \\
\text { entities }\end{array}$ & $\begin{array}{l}\text { (1) Communication relation- } \\
\text { ships (e.g., connect to) (2) } \\
\text { Dependency relationships } \\
\text { (e.g., depend on) (3) Hosting } \\
\text { relationships (e.g., hosted in) }\end{array}$ & $\begin{array}{l}\text { Entity and relation } \\
\text { specific constraints via } \\
\text { attributes }\end{array}$ & $\begin{array}{l}\text { Web based } \\
\text { GUI }\end{array}$ & DevOps \\
\hline CFEngine & $\begin{array}{l}\text { Platform } \\
\text { Resources }\end{array}$ & $\begin{array}{l}\text { Domain- } \\
\text { specific }\end{array}$ & $\begin{array}{l}\text { Support a graph } \\
\text { of resource } \\
\text { entities }\end{array}$ & $\begin{array}{l}\text { (1) Supports Dependency re- } \\
\text { lationships (e.g., depends_on) } \\
\text { (2) Support Containment } \\
\text { relationship }\end{array}$ & $\begin{array}{l}\text { Resource entity } \\
\text { specific constraints are } \\
\text { provided as attributes }\end{array}$ & $\begin{array}{l}\text { CLI, APIs, } \\
\text { Web based } \\
\text { GUI }\end{array}$ & DevOps \\
\hline Plush & $\begin{array}{l}\text { Platform } \\
\text { Resources }\end{array}$ & XML & $\begin{array}{l}\text { Support a graph } \\
\text { of resource } \\
\text { entities }\end{array}$ & $\begin{array}{l}\text { (1) Support Dependency } \\
\text { relationships (2) Support } \\
\text { Containment relationships to } \\
\text { group all the related resources }\end{array}$ & $\begin{array}{l}\text { Entity specific con- } \\
\text { straints via attributes }\end{array}$ & CLI & DevOps \\
\hline SmartFrog & $\begin{array}{l}\text { Platform } \\
\text { Resources }\end{array}$ & $\begin{array}{l}\text { Domain- } \\
\text { specific }\end{array}$ & $\begin{array}{l}\text { Support a graph } \\
\text { of resource } \\
\text { entities }\end{array}$ & $\begin{array}{l}\text { (1) Supports Inheritence and } \\
\text { Containment relationship }\end{array}$ & $\begin{array}{l}\text { Entity specific con- } \\
\text { straints via attributes }\end{array}$ & CLI & DevOps \\
\hline
\end{tabular}




\subsection{Resource Orchestration Capabilities}

Table II maps the selected orchestration techniques onto the Resource Orchestration Capabilities dimanion described in Section 5. We summarize our findings as follows:

- 7 out of 11 approaches support user-defined orchestration strategies. 4 out of 11 support rule-based orchestration. However, to the best of our knowledge none of the industry tools fully supports autonomic resources orchestration. This manifests an important need for continued research on effective, intuitive and autonomic cloud resources orchestration processes.

- We also observed, there are only a few tools such as Juju GUI, OpenTOSCA and VisualOps that provide visual abstractions to describe deployment workflows and resource topologies [Ubuntu 2013; Binz and et al. 2013; VisualOps 2015]. For example, AWS Management Console, VisualOps and CA AppLogic provide control features such as restarting, scaling and migration [VisualOps 2015]. Moreover, monitoring tools such as Nagios and CloudFielder can allow DevOps to define Service Level Agreement (SLA), detect anomalies and notify about SLA violations. Nonetheless, even then there are drawbacks amongst these approaches, in that DevOps often have to switch between multiple tools for different aspects of the management lifecycle, which proves time-consuming and cumbersome.

- We have noticed, cross-cutting concerns are reasonably addressed by research initiatives; and more so amongst enterprise-ready orchestration techniques. This is likely because their utilization in production environments require solutions that address issues such as security, portability and/or fault tolerance.

- It is apparent that various orchestration techniques employ different language paradigms. However based on our observations, there is not yet any predominant language widely adopted by the majority of cloud orchestration providers.

Accordingly, we identify the following main issues as future directions for Orchestration Capabilities:

- State-machine-based models for elasticity management. We envision state machines as a novel abstraction to dynamically represent and reason about elasticityaware resource orchestration techniques. Instead of directly manipulating lowlevel interfaces and scripting orchestration rules over complex cloud services, state machines may reason about resource requirement states. States may also characterize application-specific resource requirements (e.g., CPU and storage usages), constraints in terms of costs, and other SLAs. Transitions between states are triggered when certain conditions are satisfied (e.g., a temporal event, workload increases beyond a certain threshold). Transitions thereby automatically trigger control-actions in order to perform the desired (re-)configurations over resources to satisfy the requirements and constraints of target states.

- Visual techniques for orchestrating cloud resources. DevOps are faced with orchestrating large amounts of complex cloud resource configurations. This involves being able to proficiently understand and analyze cloud resource attributes and relationships, and make orchestration decisions on demand. We therefore believe cloud orchestration should be endowed with visual techniques to configure, deploy, monitor and control cloud resources. For example, a visual approach may allow DevOps to perform orchestration tasks. Such as, drag, drop and connect prebuilt component cloud resources; as well as deploy, monitor and manage composite cloud resources. Our previous work has introduced such a model-driven notation, based on a user-friendly and familiar mindmap interface [Weerasiri et al. 2016]. Beneath the surface, techniques are applied to manage, monitor and control cloud resource orchestrations by mapping to underlying frameworks, such as Docker. 
Table II: The Resource Orchestration Capabilities Dimension of the Selected Platforms

\begin{tabular}{|c|c|c|c|c|}
\hline & \multicolumn{4}{|c|}{ Resource Orchestration Capabilities } \\
\hline & Primitive Actions & Orchestration Strategies & Language Paradigm & Cross-cutting Concerns \\
\hline $\begin{array}{l}\text { AWS } \\
\text { OpsWorks }\end{array}$ & $\begin{array}{l}\text { Create, Delete, Describe, Update actions } \\
\text { are provided for each resource entity. } \\
\text { Clone, Start, Stop, Reboot actions are } \\
\text { offered for some entities (e.g.,- Stack, } \\
\text { Instance). Global actions are also provided. } \\
\text { (e.g., SetLoadBasedAutoScaling) }\end{array}$ & Rule-based processes & ECA rule based & $\begin{array}{l}\text { Security rules (authorization, } \\
\text { access protocols), SLA can } \\
\text { be defined via auto-scaling } \\
\text { and auto-healing rules }\end{array}$ \\
\hline $\begin{array}{l}\text { AWS } \\
\text { CloudFor- } \\
\text { mation }\end{array}$ & $\begin{array}{l}\text { Create, Delete, Update, Describe, and } \\
\text { Clone are the main actions provided }\end{array}$ & User-defined processes & Markup language & $\begin{array}{l}\text { Security rules (authorization, } \\
\text { access protocols), SLA can } \\
\text { be defined via auto-scaling } \\
\text { rules }\end{array}$ \\
\hline $\begin{array}{l}\text { VMWare } \\
\text { vSphere }\end{array}$ & $\begin{array}{l}\text { Provide a large amount of actions for each } \\
\text { entitiy type. In general all these actions can } \\
\text { be categorize into create, delete, update. }\end{array}$ & Rule-based processes & Markup language & $\begin{array}{l}\text { Security rules (authentica- } \\
\text { tion, authorization), Portable } \\
\text { VMs }\end{array}$ \\
\hline Heroku & $\begin{array}{l}\text { Create, update, scale, delete applications, } \\
\text { viewLogs (useful for monitoring) }\end{array}$ & $\begin{array}{l}\text { Autonomic and User-defined } \\
\text { processes }\end{array}$ & Script-based & $\begin{array}{l}\text { Security rules (OAuth } \\
\text { authorization) }\end{array}$ \\
\hline Puppet & Create, update and delete resources & $\begin{array}{l}\text { Mainly User-defined processes, } \\
\text { but rule-based processes for few } \\
\text { resources }\end{array}$ & $\begin{array}{l}\text { Constraint Program- } \\
\text { ming }\end{array}$ & $\begin{array}{l}\text { Security rules (encryption, } \\
\text { authentication, authorization) }\end{array}$ \\
\hline Juju & $\begin{array}{l}\text { Create and delete (Environment, VMs, and } \\
\text { Charms, Services, Relationships between } \\
\text { Charms), describe Environment, detect } \\
\text { Events, update Charm }\end{array}$ & Rule-based processes & ECA rule based & $\begin{array}{l}\text { Security rules (authentica- } \\
\text { tion, authorization) }\end{array}$ \\
\hline Docker & $\begin{array}{l}\text { Create and delete (Image, Container), share } \\
\text { (Image), start, stop, restart (Container), } \\
\text { update (Container) }\end{array}$ & User-defined processes & Script-based & $\begin{array}{l}\text { Security rules (authorization, } \\
\text { access protocols) }\end{array}$ \\
\hline $\begin{array}{l}\text { Open- } \\
\text { TOSCA }\end{array}$ & $\begin{array}{l}\text { Create, update and delete (resources and } \\
\text { relationships, attributes) }\end{array}$ & User-defined processes & Flow-based & Portable resources \\
\hline CFEngine & Create, update and delete resources & Rule-based processes & $\begin{array}{l}\text { Constraint Program- } \\
\text { ming }\end{array}$ & $\begin{array}{l}\text { Security rules (encryption, } \\
\text { authentication, authorization) }\end{array}$ \\
\hline Plush & Create (environment and application) & User-defined processes & $\begin{array}{l}\text { Markup-based and } \\
\text { Flow-based }\end{array}$ & Not addressed \\
\hline SmartFrog & Deploy, start and terminate & User-defined processes & Markup language & Not addressed \\
\hline
\end{tabular}

\subsection{Knowledge Reuse}

Table III maps the selected orchestration techniques onto the Knowledge Reuse dimensions as described in Section 8. We summarize our findings as follows:

- Research initiatives for cloud orchestration techniques generally underestimates the reuse of orchestration knowledge. Comparatively, all of the enterprise-ready approaches we analyzed, provide some form of knowledge. This observation asserts the utmost practical necessity and importance of knowledge-reuse for DevOps to build and orchestrate real-world cloud resources.

- 7 out of 11 approaches employed search indexes - the most prominent knowledge discovery technique. Amongst other search methods, keyword-based search is widely used. Generally speaking, recommendation-based knowledge discovery techniques are promising, albeit most orchestration providers do not adopt this approach due to the complexity of implementation and maintenance of the accuracy of recommendations.

- Enterprise-ready approaches predominantly support community-driven knowledge archival and curation techniques. This is due to the vast amount and diversity of cloud resources that needs to be supported. For instance, in the absence of the crowd, providers would have to build and maintain a knowledge artifact repository on their own - which would clearly be unfeasible in practice. 
The discipline of Knowledge Reuse follows a prevailing direction; namely, devising a unified representation and reuse mechanism over heterogenous artifacts, similar to what query languages offered for databases. Likewise, it is paramount to invest in a unified representation, configuration and reuse strategy over heterogenous cloud resource knowledge to enable simplified and productive cloud resources orchestration. Central to this, we prepose the concept of Orchestration Knowledge Graphs (OKGs), where common low-level orchestration logic can be abstracted, incrementally curated and thereby reused by DevOps. The type of knowledge captured can be organized into dimensions, including: Intended tasks, Resource providers, and Target environments. By identifying entities (i.e. types and attributes, relationships for each dimension, and their specialization), novel foundations will be proposed to accumulate, query and recommend currently dispersed orchestration knowledge in a structured framework.

Table III: The Knowledge Reuse Dimension of the Selected Platforms

\begin{tabular}{|c|c|c|}
\hline & \multicolumn{2}{|c|}{ Knowledge Reuse } \\
\hline & Reused Artifact & Reuse Technique \\
\hline AWS OpsWorks & Concrete and Template resource descriptions & Search index \\
\hline $\begin{array}{l}\text { AWS CloudFor- } \\
\text { mation }\end{array}$ & Concrete and Template resource descriptions & Search index \\
\hline $\begin{array}{l}\text { VMWare } \\
\text { vSphere }\end{array}$ & Portable Resource snapshots & $\begin{array}{l}\text { Search index, Recommendations, Community-driven } \\
\text { approaches (e.g., blogs) }\end{array}$ \\
\hline Heroku & Concrete and Template resource descriptions & Not specified \\
\hline Puppet & Concrete and Template resource descriptions & Community-driven search indexes \\
\hline Juju & $\begin{array}{l}\text { Concrete and Template resource descriptions and } \\
\text { Miscellaneous }\end{array}$ & Community-driven search indexes \\
\hline Docker & $\begin{array}{l}\text { Concrete and Template resource descriptions and } \\
\text { Miscellaneous }\end{array}$ & Community-driven search indexes \\
\hline OpenTOSCA & Portable Concrete and Template resource descriptions & Not specified \\
\hline CFEngine & Concrete and Template resource descriptions & Search index \\
\hline Plush & Concrete and Template resource descriptions & Not specified \\
\hline SmartFrog & Concrete and Template resource descriptions & Not specified \\
\hline
\end{tabular}

\subsection{Runtime Environment}

Table IV maps the selected orchestration techniques onto the Runtime Environment dimensions as described in Section 7. We summarize our findings as follows:

- 9 out of 11 approaches adopt a centralized execution model. This design choice is likely due to the simplicity of implementation. In comparison, decentralized orchestration requires an implementation that carefully considers discovery, synchronization, coordination and security aspects of agents.

- Surprisingly, the value of federated cloud resources is largely underestimated. Most cloud resource orchestration techniques either focus on private or public cloud environments as their target environment. Whereas, only 1 out of the 11 approaches we studied provide support for federated cloud resources management.

- The preference of virtualization technique varies largely based on the types of resources (i.e., Infrastructure, Platform or Software). All of the infrastructure focused approaches that we analyzed, adopt OS-level hypervisors as their virtualization technique. Other approaches which support Platform and Software resource adopt environment-level container managers. 
Furthermore, we identify the following future directions in the evolution of Runtime Environments:

- Runtime intelligence for autonomic and declarative orchestration. Autonomic orchestration will play a key role in addressing crucial gaps in cloud computing [Toosi et al. 2014], as well as significantly improve overall productivity. Most existing work only apply orchestration strategies for specific aspects in isolation of each other, such as configuration [Xu et al. 2012], deployment [Antonescu et al. 2013; Beloglazov et al. 2012], and control [Schulte et al. 2015].

Accordingly, We believe orchestration frameworks should be endowed with an embedded level of intelligence within their runtime environment, as well as the ability to manage themselves in accordance with high-level policies that are specified by users or administrators. For example, currently significant shortcomings exist to seamlessly integrate orchestration languages and techniques with scalable data processing platforms. In fact, such data platforms are essential for monitoring and enforcing SLAs, which involve capturing and analyzing large amounts of real-time data in big data analytics platforms (e.g., Hadoop and MapReduce). We believe the orchestration layer should contain the intelligence responsible for specifying resource orchestration, while the data processing layer should contain "the intelligence responsible for data-flow and processing" [Lemos et al. 2016].

This could be achieved by more dynamic and knowledge driven techniques that provide high-level reasoning about environment properties and automated support for policies provisioning to support a range of autonomic orchestration tasks such as self-configuration and self-optimization; as well as self-healing and selfprotecting tasks. DevOps will thus be able to describe resource requirements and constraints using declarative and orchestration-aware abstractions such as State machines (refer to Section 9.3). Orchestration runtimes may thus automatically translate such abstractions into efficient and technique-aware execution scripts.

- Cloud service event summaries. The ability for cloud orchestration platforms to gain the requisite intelligence about consumption patterns of deployed resources, ensures compliance with cost and SLA constraints, and improves resource orchestration processes in general (e.g., continuously fine-tuning defined policies in dynamic and evolving environments).

We therefore believe future work should develop concepts and techniques to model and capture event patterns and abstract them into meaningful concepts (e.g., characterizing states of an application or a service, state of a specific application component, behavior of users from a specific geolocation) that are suitable for cloud elastic resource orchestration purposes. Accordingly, we believe high-level language constructs to abstract and aggregate temporal and resource-relevant events over federated cloud services at various granularities will provide the key. These can be used to describe event summaries of knowledge about variations in resource requirements, in terms of both aggregated resource consumption metrics (e.g., the number of API calls per second) and semantically meaningful event categories (e.g., moderate application load). Event summaries can be defined at various abstraction levels as a hierarchy to cater for context-based, fine- or coarsegrained analysis of resource requirements and consumption trends. Lower-level event summaries may be concrete (e.g., providing knowledge relevant to a finegrain analysis of patterns for some specific cloud service such as Amazon DynamoDB). Higher-level event summaries may capture knowledge required for coarse-grain analysis of patterns relevant to a collection of resources (e.g., cluster, whole application). 
Table IV: The Runtime Environment Dimension of the Selected Platforms

\begin{tabular}{|c|c|c|c|}
\hline & \multicolumn{3}{|l|}{ Runtime Environment } \\
\hline & Virtualization Technique & Execution Model & Target Environment \\
\hline $\begin{array}{l}\text { AWS } \\
\text { OpsWorks }\end{array}$ & OS-level hypervisor & Centralized & Public Cloud \\
\hline $\begin{array}{l}\text { AWS } \\
\text { OpsWorks }\end{array}$ & OS-level hypervisor & Centralized & Public Cloud \\
\hline $\begin{array}{l}\text { VMWare } \\
\text { vSphere }\end{array}$ & OS-level hypervisor & Centralized & Private Cloud \\
\hline Heroku & Environment-level Container manager & Centralized & Public Cloud \\
\hline Puppet & $\begin{array}{l}\text { Not relevant (only responsible for configuration management of resources rather } \\
\text { virtualizing them) }\end{array}$ & De-centralized & Public or Private Cloud \\
\hline Juju & OS-level hypervisor & Centralized & Public or Private Cloud \\
\hline Docker & Environment-level Container manager & Centralized & Public or Private Cloud \\
\hline $\begin{array}{l}\text { Open- } \\
\text { TOSCA }\end{array}$ & OS-level hypervisor & Centralized & Public or Private Cloud \\
\hline CFEngine & $\begin{array}{l}\text { Not relevant (only responsible for configuration management of resources rather } \\
\text { virtualizing them) }\end{array}$ & De-centralized & Public or Private Cloud \\
\hline Plush & $\begin{array}{l}\text { Not relevant (only responsible for configuration management of resources rather } \\
\text { virtualizing them) }\end{array}$ & Centralized & Private Cloud \\
\hline SmartFrog & $\begin{array}{l}\text { Not relevant (only responsible for configuration management of resources rather } \\
\text { virtualizing them) }\end{array}$ & Centralized & Private Cloud \\
\hline
\end{tabular}

\section{CONCLUSION}

Cloud resources and orchestration techniques are an effective technology, endowed with immense power to transform traditional infrastructure, platform and software resources into elastic, measurable, on-demand self-service-based virtual components. In this extensive survey, we have studied a diverse mix of cloud resource orchestration techniques, which include languages, services, standards, and tools. We presented a novel taxonomy over a broad range of relevant dimensions, which we have applied to characterize and analyse various orchestration techniques. We contribute a systematic analysis of the most representative cloud resource orchestration techniques by evaluating and classifying them against the presented taxonomy. Towards the end of this contribution, we derive key open research issues based on the apparent technical gaps that were identified during the analysis. Accordingly, we propose a range of future directions as fruitful guidelines for the next generation of cloud orchestration.

\section{REFERENCES}

Brian Adler. 2011. Building Scalable Applications In the Cloud: Reference Architecture \& Best Practices, RightScale Inc. (2011). https://s3.amazonaws.com/aws001/guided_trek/RightScale_White_Paper Building_Scalable_Applications.pdf

Jeannie Albrecht and et al. 2011. Distributed application configuration, management, and visualization with plush. ACM Transactions on Internet Technology (TOIT) 11, 2 (2011), 6.

Khalid Alhamazani, Rajiv Ranjan, Karan Mitra, Fethi Rabhi, Prem Prakash Jayaraman, Samee Ullah Khan, Adnene Guabtni, and Vasudha Bhatnagar. 2015. An overview of the commercial cloud monitoring tools: research dimensions, design issues, and state-of-the-art. Computing 97, 4 (2015), 357-377.

AWS Amazon. 2011. AWS Cloud Formation. Online article. (2011). Retrieved May 7, 2015 from http://aws. amazon.com/cloudformation/

AWS Amazon. 2015. AWS OpsWorks Template Snippets. Online article. (2015). Retrieved June 24, 2015 from http://docs.aws.amazon.com/AWSCloudFormation/latest/UserGuide/quickref-opsworks.html

Inc Amazon Web Services. 2015a. Amazon EC2 Instances. Online article. (2015). Retrieved June 8, 2015 from http://aws.amazon.com/ec2/instance-types/ 
Inc. Amazon Web Services. 2015b. Amazon Relational Database Service - API Docuumentation. Online article. (2015). Retrieved June 8, 2015 from http://docs.aws.amazon.com/AmazonRDS/latest/APIReference/ Welcome.html

Inc. Amazon Web Services. 2015c. AWS SDK for Java. Online article. (2015). Retrieved November 10, 2015 from https://aws.amazon.com/sdk-for-java/

Inc. Amazon Web Services. 2015d. REST API for AWS S3. Online article. (2015). Retrieved November 10, 2015 from http://docs.aws.amazon.com/AmazonS3/latest/API/APIRest.html

Inc. Ansible. 2015. Ansible : Cloud Modules. Online article. (2015). Retrieved June 10, 2015 from http: //docs.ansible.com/list_of_cloud_modules.html

Alexandru-Florian Antonescu, Alvaro Gomes, Peter Robinson, and Torsten Braun. 2013. SLA-driven predictive orchestration for distributed cloud-based mobile services. In Communications Workshops (ICC), 2013 IEEE International Conference on. IEEE, 738-743.

CA AppLogic. 2015. CA AppLogic Cloud Platform. Online article. (2015). Retrieved May 28, 2015 from http: //www.ca.com/us/products/detail/ca-applogic.aspx

Claudio A. Ardagna, Rasool Asal, Ernesto Damiani, and Quang Hieu Vu. 2015. From Security to Assurance in the Cloud: A Survey. ACM Comput. Surv. 48, 1, Article 2 (July 2015), 50 pages. DOI : http://dx.doi.org/10.1145/2767005

D. Ardagna and et al. 2012. MODAClouds: A model-driven approach for the design and execution of applications on multiple Clouds. In MISE, 2012 ICSE Workshop on. 50-56. DOI : http://dx.doi.org/10.1109/MISE.2012.6226014

Michael Armbrust and et al. 2010. A View of Cloud Computing. Commun. ACM 53, 4 (April 2010), 50-58. DOI : http://dx.doi.org/10.1145/1721654.1721672

Amazon Auto Scaling. 2015. Auto Scaling for AWS cloud resources. Online article. (2015). Retrieved May 7, 2015 from http://aws.amazon.com/autoscaling/

AWS. 2013a. Available commands for EC2 in AWS CLI. Online article. (2013). Retrieved May 7, 2015 from http://docs.aws.amazon.com/cli/latest/reference/ec2/index.html

AWS. 2013b. AWS CLI. Online article. (2013). Retrieved May 7, 2015 from http://docs.aws.amazon.com/cli/ latest/index.html

Arshdeep Bahga and Vijay K Madisetti. 2013. Rapid Prototyping of Multitier Cloud-Based Services and Systems. Computer 46, 11 (2013), 76-83.

Wolfgang Barth. 2008. Nagios: System and network monitoring. No Starch Press.

Erick Bauman, Gbadebo Ayoade, and Zhiqiang Lin. 2015. A Survey on Hypervisor-Based Monitoring: Approaches, Applications, and Evolutions. ACM Comput. Surv. 48, 1, Article 10 (Aug. 2015), 33 pages. DOI : http://dx.doi.org/10.1145/2775111

Anton Beloglazov, Jemal Abawajy, and Rajkumar Buyya. 2012. Energy-aware resource allocation heuristics for efficient management of data centers for cloud computing. Future generation computer systems 28,5 (2012), 755-768.

Anton Beloglazov, Rajkumar Buyya, Young Choon Lee, Albert Zomaya, and others. 2011. A taxonomy and survey of energy-efficient data centers and cloud computing systems. Advances in computers 82, 2 (2011), 47-111.

Alexander Bergmayr, Alessandro Rossini, Nicolas Ferry, Geir Horn, Leire Orue-Echevarria, Arnor Solberg, and Manuel Wimmer. 2015. The Evolution of CloudML and its Applications. In Proceedings of the 3rd International Workshop on Model-Driven Engineering on and for the Cloud 18th International Conference on Model Driven Engineering Languages and Systems (MoDELS 2015), Ottawa, Canada, September 29, 2015. 13-18. http://ceur-ws.org/Vol-1563/paper3.pdf

Jan A. Bergstra and Mark Burgess. 2008. A static theory of promises. CoRR abs/0810.3294 (2008). http: //arxiv.org/abs/0810.3294

Jan A Bergstra and Mark Burgess. 2014. Promises, Impositions, and other Directionals. arXiv preprint arXiv:1401.3381 (2014).

Tobias Binz and et al. 2013. OpenTOSCA-a runtime for TOSCA-based cloud applications. In ServiceOriented Computing. Springer, 692-695.

Bitnami. 2015. Bitnami makes it easy to run your favorite server apps anywhere. Online article. (2015). Retrieved May 28, 2015 from https://bitnami.com/learn_more

Thomas J. Bittman. 2011. The Road Map From Virtualization to Cloud Computing. https://www.gartner. com/doc/1572031. (March 2011). Accessed: 24/11/2013.

Paul Borril, Mark Burgess, Todd Craw, and Mike Dvorkin. 2014. A promise theory perspective on data networks. arXiv preprint arXiv:1405.2627 (2014).

Mark Burgess. 2007. Promise you a rose garden. (2007). 
Mark Burgess. 2009. Knowledge management and promises. In Scalability of Networks and Services. Springer, 95-107.

Mark Burgess. 2011. Testable system administration. Commun. ACM 54, 3 (2011), 44-49.

Mark Burgess and Oslo College. 1995. Cfengine: a site configuration engine. In in USENIX Computing systems, Vol.

Mark Burgess and Alva L Couch. 2006. Modeling Next Generation Configuration Management Tools.. In LISA. 131-147.

Damon Cali. 2013. Introducing rumm: a Command Line Tool for the Rackspace Cloud. Online article. (2013). Retrieved June 9, 2015 from https://developer.rackspace.com/blog/ introducing-rumm-a-command-line-tool-for-the-rackspace-cloud/

Canonical. 2015. Juju Charm Store. Online article. (2015). Retrieved September 8, 2015 from https: //jujucharms.com/store

CenturyLink. 2015. Panamax: Docker Management for Humans. Online article. (2015). Retrieved November 10, 2015 from http://panamax.io/

Clovis Chapman, Wolfgang Emmerich, Fermín Galán Márquez, Stuart Clayman, and Alex Galis. 2012. Software architecture definition for on-demand cloud provisioning. Cluster Computing 15, 2 (2012), 79-100.

Muhammad Aufeef Chauhan, Muhammad Ali Babar, and Boualem Benatallah. 2016. Architecting cloudenabled systems: a systematic survey of challenges and solutions. Software: Practice and Experience (2016).

Chef. 2015. About Recipes. Online article. (2015). https://docs.chef.io/recipes.html

Peter Pin-Shan Chen. 1976. The Entity-relationship Model\&Mdash;Toward a Unified View of Data. ACM Trans. Database Syst. 1, 1 (March 1976), 9-36. DOI : http://dx.doi.org/10.1145/320434.320440

Shang-Wen Cheng and David Garlan. 2012. Stitch: A Language for Architecture-based Self-adaptation. J. Syst. Softw. 85, 12 (Dec. 2012), 2860-2875. DOI : http://dx.doi.org/10.1016/j.jss.2012.02.060

Trieu C Chieu and at al. 2010. Solution-based deployment of complex application services on a cloud. In SOLI, 2010 IEEE International Conference on. IEEE, 282-287.

Mark Chignell, James Cordy, Joanna Ng, and Yelena Yesha. 2010. The smart internet: current research and future applications. Vol. 6400. Springer Science \& Business Media.

Cisco-Systems-Inc. 2011. Cloud: what an enterprise must know. Online article. (2011). Retrieved December 2011 from http://www.cisco.com/en/US/solutions/collateral/ns340/ns517/ns224/ns836/ns976/white_ paper_c11-617239.pdf

Cloud-Foundry. 2016. The industry standard platform for cloud applications. Online article. (2016). Retrieved June 5, 2016 from https://www.cloudfoundry.org/

Inc. CloudBees. 2016. CloudBees: The Enterprise Jenkins Company. Online article. (2016). Retrieved January 10, 2016 from https://www.cloudbees.com/

Apache CloudStack. 2016. Apache cloudstack: Open source cloud computing. Online article. (2016). Retrieved January 10, 2016 from https:/cloudstack.apache.org/

AWS CloudTrail. 2014. Security at scale: Logging in AWS. (2014).

Amazon CloudWatch. 2013. Monitoring for AWS cloud resources. Online article. (2013). Retrieved May 7, 2015 from http://aws.amazon.com/cloudwatch/

Alva L Couch, John Hart, Elizabeth G Idhaw, and Dominic Kallas. 2003. Seeking Closure in an Open World: A Behavioral Agent Approach to Configuration Management.. In LISA, Vol. 3. 125-148.

S. Crosby and et al. 2009. Open virtualization format specification. Standards and Technology, no. DSP0243 in DMTF Specifications, Distributed Management Task Force (2009).

CS50. 2015. CS50 Appliance 19. Online article. (2015). Retrieved June 8, 2015 from https://manual.cs50.net/ appliance/19/

CSA. 2011. Security guidance for critical areas of focus in cloud computing. Online article. (2011). Retrieved November 2011 from https://cloudsecurityalliance.org/research/securityguidance/

Yong Cui, Vojislav B Misic, Rajkumar Buyya, and Dejan Milojicic. 2013. Guest Editors' Introduction: Special Issue on Cloud Computing. IEEE Transactions on Parallel and Distributed Systems 24, 6 (2013).

Michael Cusumano. 2010. Cloud computing and SaaS as new computing platforms. Commun. ACM 53, 4 (2010), 27-29.

Clemens Danninger. 2015. Using Constraint Solvers to Find Valid Software Configurations. (2015).

James Darvell. 2016. Unikernels, Docker, and Why You Should Care. Online article. (2016). Retrieved November 25, 2016 from http://www.linuxjournal.com/content/ unikernels-docker-and-why-you-should-care/ 
Thomas Delaet, Wouter Joosen, and Bart Vanbrabant. 2010. A Survey of System Configuration Tools. In Proceedings of the 24th International Conference on LISA. USENIX Association, 1-8. http://dl.acm.org/ citation.cfm?id=1924976.1924977

Hewlett Packard Enterprise Development. 2016. HPE Helion Eucalyptus: Open source hybrid cloud software for AWS users. Online article. (2016). Retrieved January 10, 2016 from http://www8.hp.com/us/en/ cloud/helion-eucalyptus-overview.html

Zuohua Ding, Yuan Zhou, and MengChu Zhou. 2014. Modeling self-adaptive software systems with learning petri nets. In Companion Proceedings of the 36th International Conference on Software Engineering. ACM, 464-467.

Nectar Directorate. 2016. Nectar: Australia's fastest growing researcher network. Online article. (2016). Retrieved January 10, 2016 from https://nectar.org.au/

DMTF. 2010. Architecture for managing clouds - A white paper from the open cloud standards incubator. Online article. (2010). Retrieved June 2010 from http://dmtf.org/standards/cloud/

Docker. 2015a. Docker Hub Registry. Online article. (2015). https://registry.hub.docker.com/

Docker. 2015b. Overview of Docker Compose. Online article. (2015). https://docs.docker.com/compose/

dotCloud. 2015. Online article. (2015). https://www.dotcloud.com/dev-center/platform-documentation

Robert Dukaric and Matjaz B Juric. 2013. Towards a unified taxonomy and architecture of cloud frameworks. Future Generation Computer Systems 29, 5 (2013), 1196-1210.

Marlon Dumas, Marcello La Rosa, Jan Mendling, and Hajo A Reijers. 2013. Fundamentals of business process management. Springer.

Erik Elmroth and Lars Larsson. 2009. Interfaces for placement, migration, and monitoring of virtual machines in federated clouds. In GCC'09. IEEE, 253-260.

Inc. Engine Yard. 2016. Engine Yard. Online article. (2016). Retrieved January 10, 2016 from https://www. engineyard.com/

Daren Fang, Xiaodong Liu, Imed Romdhani, and Claus Pahl. 2015. An approach to unified cloud service access, manipulation and dynamic orchestration via semantic cloud service operation specification framework. Journal of Cloud Computing 4, 1 (2015), 1.

Wei Fang, ZhiHui Lu, Jie Wu, and ZhenYin Cao. 2012. RPPS: a novel resource prediction and provisioning scheme in cloud data center. In Services Computing (SCC), 2012 IEEE Ninth International Conference on. IEEE, 609-616.

Kaniz Fatema, Vincent C Emeakaroha, Philip D Healy, John P Morrison, and Theo Lynn. 2014. A survey of Cloud monitoring tools: Taxonomy, capabilities and objectives. J. Parallel and Distrib. Comput. 74, 10 (2014), 2918-2933.

Finally.io. 2014. finally.io. Online article. (2014). Retrieved February 8, 2015 from https://www.finally.io/

Linux Foundation. 2015a. Ooen Container Project. Online article. (2015). Retrieved June 24, 2015 from http://www.opencontainers.org/

Linux Foundation. 2015b. OPEN CONTAINER INITIATIVE. https://www.opencontainers.org/. (2015). Accessed: $24 / 09 / 2015$.

The Apache Software Foundation. 2014a. An API that abstracts the differents between clouds. Online article. (2014). Retrieved June 10, 2015 from https://deltacloud.apache.org/

The Apache Software Foundation. 2014b. Compute Guide. Online article. (2014). Retrieved November 10, 2015 from https://jclouds.apache.org/start/compute/

The Apache Software Foundation. 2014c. The Java Multi-Cloud Toolkit. Online article. (2014). Retrieved June 10, 2015 from https://jclouds.apache.org/

The Apache Software Foundation. 2015c. One Interface To Rule Them All. Online article. (2015). Retrieved June 10, 2015 from https://libcloud.apache.org/

Joerg Fritsch. 2015. Security properties of Containers managed by Docker. https://www.gartner.com/doc/ 2956826/security-properties-containers-managed-docker. (January 2015). Accessed: 05/06/2015.

Gartner. 2013. Gartner Says Cloud Computing Will Become the Bulk of New IT Spend by 2016. http://www. gartner.com/newsroom/id/2613015. (October 2013). Accessed: 24/11/2015.

Inc. Gartner. 2014. Gartner Survey Reveals That SaaS Deployments Are Now Mission Critical. http://www. gartner.com/newsroom/id/2923217. (November 2014). Accessed: 14/07/2015.

Wolfgang Gerlach and et al. 2014. Skyport: container-based execution environment management for multicloud scientific workflows. In Proceedings of the 5th International Workshop on Data-Intensive Computing in the Clouds. IEEE Press, 25-32.

Patrick Goldsack and at al. 2009. The SmartFrog configuration management framework. ACM SIGOPS Operating Systems Review 43, 1 (2009), 16-25. 
Patrick Goldsack, Julio Guijarro, Steve Loughran, Alistair N. Coles, Andrew Farrell, Antonio Lain, Paul Murray, and Peter Toft. 2009. The SmartFrog configuration management framework. Operating Systems Review 43, 1 (2009), 16-25.

Google. 2015a. CONTAINER REGISTRY: Fast, private Docker image storage on Google Cloud Platform. Online article. (2015). Retrieved November 17, 2015 from https://cloud.google.com/container-registry/

Google. 2015b. Google App Engine: Platform as a Service. Online article. (2015). Retrieved June 8, 2015 from https://cloud.google.com/appengine/docs

Christophe Gravier and et al. 2013. Context awareness as a Service for Cloud Resource Optimization. (2013).

Christophe Gravier, Julien Subercaze, Amro Najjar, Frederique Laforest, Xavier Serpaggi, and Olivier Boissier. 2015. Context Awareness as a Service for Cloud Resource Optimization. Internet Computing, IEEE 19, 1 (2015), 28-34.

Diwaker Gupta, Ludmila Cherkasova, Rob Gardner, and Amin Vahdat. 2006. Enforcing Performance Isolation Across Virtual Machines in Xen. In Proceedings of the ACM International Conference on Middleware (Middleware '06). Springer-Verlag New York, Inc., New York, NY, USA, 342-362.

Mohammad Hajjat, Xin Sun, Yu-Wei Eric Sung, David Maltz, Sanjay Rao, Kunwadee Sripanidkulchai, and Mohit Tawarmalani. 2011. Cloudward bound: planning for beneficial migration of enterprise applications to the cloud. ACM SIGCOMM Computer Communication Review 41, 4 (2011), 243-254.

Abdul Hameed, Alireza Khoshkbarforoushha, Rajiv Ranjan, Prem Prakash Jayaraman, Joanna Kolodziej, Pavan Balaji, Sherali Zeadally, Qutaibah Marwan Malluhi, Nikos Tziritas, Abhinav Vishnu, and others. 2014. A survey and taxonomy on energy efficient resource allocation techniques for cloud computing systems. Computing (2014), 1-24.

Ahmad Fadzil M. Hani, Irving Vitra Paputungan, and Mohd Fadzil Hassan. 2015. Renegotiation in Service Level Agreement Management for a Cloud-Based System. ACM Comput. Surv. 47, 3, Article 51 (April 2015), 21 pages. DOI : http://dx.doi.org/10.1145/2716319

Mitchell Hashimoto. 2013. Vagrant: Up and Running. O'Reilly Media, Inc.

Christina N Hoefer and Georgios Karagiannis. 2010. Taxonomy of cloud computing services. In 2010 IEEE Globecom Workshops. IEEE, 1345-1350.

Ben Hosmer. 2012. Getting started with salt stack-the other configuration management system built with python. Linux journal 2012, 223 (2012), 3.

Wei Huang and et al. 2015. The State of Public Infrastructure-as-a-Service Cloud Security. ACM Comput. Surv. 47, 4, Article 68 (June 2015), 31 pages. DOI : http://dx.doi.org/10.1145/2767181

Cloudlabs Inc. 2015. Public Snaps. Online article. (2015). Retrieved November 17, 2015 from https://www. terminal.com/explore

TIBCO Software Inc. 2014. Event Processing with State Machines. Technical Report.

Intel-Corporation. 2015. Cloud computing taxonomy and ecosystem analysis. Online article. (2015). Retrieved September 2012 from http:/www.intel.com/content/dam/doc/case-study/ intel-it-cloudcomputing-taxonomy-ecosystem-analysis-study.pdf

Waheed Iqbal, Matthew N Dailey, David Carrera, and Paul Janecek. 2011. Adaptive resource provisioning for read intensive multi-tier applications in the cloud. Future Generation Computer Systems 27, 6 (2011), 871-879.

Sadeka Islam, Jacky Keung, Kevin Lee, and Anna Liu. 2012. Empirical prediction models for adaptive resource provisioning in the cloud. Future Generation Computer Systems 28, 1 (2012), 155-162.

Brendan Jennings and Rolf Stadler. 2014. Resource management in clouds: Survey and research challenges. Journal of Network and Systems Management (2014), 1-53.

Yexi Jiang, Chang-shing Perng, Tao Li, and Rong Chang. 2011. Asap: A self-adaptive prediction system for instant cloud resource demand provisioning. In Data Mining (ICDM), 2011 IEEE 11th International Conference on. IEEE, 1104-1109.

Ubuntu Juju. 2015a. Charm Store Policy. Online article. (2015). Retrieved June 8, 2015 from https:/juju. ubuntu.com/docs/authors-charm-policy.html

Ubuntu Juju. 2015b. What is a relation? Online article. (2015). Retrieved June 8, 2015 from https: //jujucharms.com/docs/stable/authors-interfaces

Matjaz B Juric and Denis Weerasiri. 2014. WS-BPEL 2.0 beginner's guide. Packt Publishing Ltd.

Eleni Kamateri, Nikolaos Loutas, Dimitris Zeginis, James Ahtes, Francesco D’Andria, Stefano Bocconi, Panagiotis Gouvas, Giannis Ledakis, Franco Ravagli, Oleksandr Lobunets, and others. 2013. Cloud4soa: A semantic-interoperability paas solution for multi-cloud platform management and portability. In European Conference on Service-Oriented and Cloud Computing. Springer, 64-78.

Luke Kanies. 2006. Puppet: Next-generation configuration management. The USENIX Magazine 31, 1 (2006), 19-25. 
B Khasnabish, J Chu, S Ma, Y Meng, N So, P Unbehagen, and others. 2011. IEFT cloud reference framework. (2011). http://tools.ietf.org/html/draft-khasnabishcloud-reference-framework-02

Alireza Khoshkbarforoushha, Meisong Wang, Rajiv Ranjan, Lizhe Wang, Leila Alem, Samee U Khan, and Boualem Benatallah. 2016. Dimensions for Evaluating Cloud Resource Orchestration Frameworks. Computer 49, 2 (2016), 24-33.

Hyunjoo Kim and Manish Parashar. 2011. CometCloud: An autonomic cloud engine. Cloud Computing: Principles and Paradigms (2011), 275-297.

Johannes Kirschnick and et al. 2012. Towards an Architecture for Deploying Elastic Services in the Cloud. Softw. Pract. Exper. 42, 4 (April 2012), 395-408. DOI: http://dx.doi.org/10.1002/spe.1090

Alexander V. Konstantinou and et al. 2009. An Architecture for Virtual Solution Composition and Deployment in Infrastructure Clouds. In Proceedings of the 3rd International Workshop on VTDC. ACM, 9-18.

Oliver Kopp and et al. 2012. BPMN4TOSCA: A domain-specific language to model management plans for composite applications. In Business Process Model and Notation. Springer, 38-52.

Puppet Labs. 2015a. Overview of Orchestration Topics. Online article. (2015). Retrieved October 10, 2015 from https://docs.puppetlabs.com/pe/latest/orchestration_overview.html

Puppet Labs. 2015b. Publishing Modules on the Puppet Forge. Online article. (2015). Retrieved June 8, 2015 from https://docs.puppetlabs.com/puppet/latest/reference/modules_publishing.html

Puppet Labs. 2015c. Puppet Enterprise. Online article. (2015). Retrieved October 10, 2015 from https:// puppetlabs.com/puppet/puppet-enterprise

Puppet Labs. 2015d. Type Reference. Online article. (2015). Retrieved June 8, 2015 from https://docs. puppetlabs.com/references/latest/type.html

Peter Laird. 2008. Cloud Taxonomy. Online article. (2008). Retrieved September 2008 from https://sites. google.com/site/saaslink/Laird_CloudMap_Sept2008.png

C. Larman and V.R. Basili. 2003. Iterative and incremental developments. a brief history. Computer 36, 6 (June 2003), 47-56. DOI : http://dx.doi.org/10.1109/MC.2003.1204375

George Lawton. 2005. LAMP lights enterprise development efforts. Computer 38, 9 (2005), 0018-20.

Angel Lagares Lemos, Florian Daniel, and Boualem Benatallah. 2016. Web service composition: a survey of techniques and tools. ACM Computing Surveys (CSUR) 48, 3 (2016), 33.

Grace Lewis and others. 2013. Role of standards in cloud-computing interoperability. In System Sciences (HICSS), 2013 46th Hawaii International Conference on. IEEE, 1652-1661.

Christoph Fehling Frank Leymann, Ralph Retter, Walter Schupeck, and Peter Arbitter. 2014. Cloud computing patterns. (2014).

LinuxContainers.org. 2015. What's LXC? Online article. (2015). Retrieved June 8, 2015 from https:// linuxcontainers.org/lxc/introduction/

Changbin Liu, Boon Thau Loo, and Yun Mao. 2011a. Declarative Automated Cloud Resource Orchestration. In Proceedings of the SOCC'11. ACM, Article 26, 8 pages.

Changbin Liu, Yun Mao, Jacobus Van der Merwe, and Mary Fernandez. 2011b. Cloud resource orchestration: A data-centric approach. In Proceedings of the biennial Conference on Innovative Data Systems Research (CIDR). 1-8.

Fang Liu, Jin Tong, Jian Mao, Robert Bohn, John Messina, Lee Badger, and Dawn Leaf. 2011c. NIST cloud computing reference architecture. NIST special publication 500, 2011 (2011), 292.

Scott Lowe. 2011. Mastering VMware vSphere 5. John Wiley \& Sons.

Real Status Ltd. 2015. A visibly different approach to cross-domain, hybrid IT management. Online article. (2015). Retrieved October 7, 2015 from http:/www.hyperglance.com/wp-content/uploads/2015/08/ HyperglanceDatasheet_Final1.pdf

Hongbin Lu, M. Shtern, B. Simmons, M. Smit, and M. Litoiu. 2013. Pattern-Based Deployment Service for Next Generation Clouds. In Services (SERVICES), 2013 IEEE Ninth World Congress on. 464-471.

Heiko Ludwig, Alexander Keller, Asit Dan, Richard King, and Richard Franck. 2003. A service level agreement language for dynamic electronic services. Electronic Commerce Research 3, 1-2 (2003), 43-59.

MadeiraCloud. 2015. CloudFielder: Policy as a Service, for your cloud infrastrucutre. Online article. (2015). Retrieved October 10, 2015 from http://cloudfielder.com/

David J. Malan. 2015. CS50. Online article. (2015). Retrieved June 8, 2015 from https://cs50.harvard.edu/

Ebrahim H Mamdani. 1974. Application of fuzzy algorithms for control of simple dynamic plant. In Proceedings of the Institution of Electrical Engineers, Vol. 121. IET, 1585-1588.

Zoltán Ádám Mann. 2015. Allocation of Virtual Machines in Cloud Data Centers-A Survey of Problem Models and Optimization Algorithms. ACM Comput. Surv. 48, 1, Article 11 (Aug. 2015), 34 pages. 
Amazon Marketplace. 2012. Marketplace for AWS cloud resources. Online article. (2012). Retrieved May 7, 2015 from https:/aws.amazon.com/marketplace

Toni Mastelic, Ariel Oleksiak, Holger Claussen, Ivona Brandic, Jean-Marc Pierson, and Athanasios V. Vasilakos. 2014. Cloud Computing: Survey on Energy Efficiency. ACM Comput. Surv. 47, 2, Article 33 (Dec. 2014), 36 pages. DOI : http://dx.doi.org/10.1145/2656204

Michael Menzel, Rajiv Ranjan, Lizhe Wang, Samee U Khan, and Jinjun Chen. 2015. CloudGenius: a hybrid decision support method for automating the migration of web application clusters to public clouds. Computers, IEEE Transactions on 64, 5 (2015), 1336-1348.

Thijs Metsch, Andy Edmonds, R Nyrén, and A Papaspyrou. 2010. Open cloud computing interface-core. In Open Grid Forum, OCCI-WG, Specification Document.

Brenda M Michelson. 2006. Event-driven architecture overview. Patricia Seybold Group 2 (2006).

Neil Middleton, Richard Schneeman, and others. 2013. Heroku: Up and Running. O'Reilly Media, Inc.

M. Mishra, A. Das, P. Kulkarni, and A. Sahoo. 2012. Dynamic resource management using virtual machine migrations. Communications Magazine, IEEE 50, 9 (September 2012), 34-40.

Madhurranjan Mohaan and Ramesh Raithatha. 2014. Learning Ansible. Packt Publishing Ltd.

Francesco Moscato and et al. 2011. An analysis of mOSAIC ontology for Cloud resources annotation. In FedCSIS, 2011. IEEE, 973-980.

Cohesive Networks. 2016. Cohesive Networks: Home. Online article. (2016). https://cohesive.net/

Nitrous. 2013. nitrous.io. Online article. (2013). Retrieved May 7, 2015 from https://nitrous.io

OASIS 2013. Topology and Orchestration Specification for Cloud Applications (TOSCA), Version 1.0. OASIS.

Kiran Oliver. 2015. TNS Markers: The Comparison and Context of Unikernels and Containers. Online article. (2015). Retrieved November 25, 2016 from http:/thenewstack.io/ the-comparison-and-context-of-unikernels-and-containers/

OMG 2011. Business Process Model and Notation (BPMN), Version 2.0. OMG.

OpenCrowd. 2010. Cloud Taxonomy. (2010). http://cloudtaxonomy.opencrowd.com

OpenStack.org. 2015a. Open source software for creating private and public clouds. Online article. (2015). Retrieved May 30, 2015 from https://www.openstack.org/

OpenStack.org. 2015b. OpenStack Orchestration. Online article. (2015). Retrieved June 10, 2015 from https: //wiki.openstack.org/wiki/Heat

Oracle-Corporation. 2011. Oracle reference architecture-cloud infrastructure. Online article. (2011). Retrieved November 2011 from http://www.oracle.com/technetwork/topics/entarch/ oracle-ra-cloudinfrastructure-r3-0-1395892.pdf

Suraj Pandey, Linlin Wu, Siddeswara Mayura Guru, and Rajkumar Buyya. 2010. A particle swarm optimization-based heuristic for scheduling workflow applications in cloud computing environments. In Advanced Information Networking and Applications (AINA), 2010 24th IEEE International Conference on. IEEE, 400-407.

Manish Parashar and Salim Hariri. 2005. Autonomic computing: An overview. In Unconventional Programming Paradigms. Springer, 257-269.

Google Cloud Platform. 2015. Cloud SDK. Online article. (2015). Retrieved May 9, 2015 from https://cloud. google.com/sdk/

OpenNebula Project. 2016. OpenNebula - Flexible Enterprise Cloud Made Simple. Online article. (2016). Retrieved January 10, 2016 from http://opennebula.org/

Rackspace. 2015. Rackspace: API Documentation. Online article. (2015). http://docs.rackspace.com/

Fahimeh Ramezani, Jie Lu, and Faheem Hussain. 2013. An online fuzzy decision support system for resource management in cloud environments. In IFSA World Congress and NAFIPS Annual Meeting (IFSA/NAFIPS), 2013 Joint. IEEE, 754-759.

Rajiv Ranjan, Boualem Benatallah, Schahram Dustdar, and Michael P Papazoglou. 2015. Cloud Resource Orchestration Programming: Overview, Issues, and Directions. IEEE Internet Computing 19, 5 (2015), 46-56.

Rajiv Ranjan, Rajkumar Buyya, and Surya Nepal. 2013. Editorial: Model-driven Provisioning of Application Services in Hybrid Computing Environments. Future Gener. Comput. Syst. 29, 5 (July 2013), 1211-1215. DOI : http://dx.doi.org/10.1016/j.future.2013.01.007

Paul Resnick and Hal R Varian. 1997. Recommender systems. Commun. ACM 40, 3 (1997), 56-58.

Stefan Ried, Holger Kisker, and Pascal Matzke. 2010. The evolution of cloud computing markets. Forrester Research (2010).

RightScale. 2016. Self-Service. Online article. (2016). Retrieved April 23, 2016 from http://www.rightscale. com/products-and-services/products/self-service 
Bhaskar Prasad Rimal, Eunmi Choi, and Ian Lumb. 2009. A taxonomy and survey of cloud computing systems. INC, IMS and IDC (2009), 44-51.

Rami Rosen. 2013. Resource management: Linux kernel Namespaces and cgroups. Haifux, May (2013).

Todd Rosner. 2013. Learning AWS OpsWorks. Packt Publishing Ltd.

Arpan Roy, Santonu Sarkar, Rajeshwari Ganesan, and Geetika Goel. 2015. Secure the Cloud: From the Perspective of a Service-Oriented Organization. ACM Comput. Surv. 47, 3, Article 41 (Feb. 2015), 30 pages. DOI : http://dx.doi.org/10.1145/2693841

Navin Sabharwal. 2014. Automation Through Chef Opscode. APress.

H. Sato, A. Kanai, and S. Tanimoto. 2010. A Cloud Trust Model in a Security Aware Cloud. In Applications and the Internet (SAINT), 2010 10th IEEE / IPSJ International Symposium on. 121-124.

Benjamin Satzger and et al. 2013. Winds of Change: From Vendor Lock-In to the Meta Cloud. Internet Computing, IEEE 17, 1 (2013), 69-73.

Pete Sawyer, Raul Mazo, Daniel Diaz, Camille Salinesi, and Danny Hughes. 2012. Using constraint programming to manage configurations in self-adaptive systems. Computer 10 (2012), 56-63.

Stefan Schulte, Christian Janiesch, Srikumar Venugopal, Ingo Weber, and Philipp Hoenisch. 2015. Elastic Business Process Management: State of the art and open challenges for BPM in the cloud. Future Generation Computer Systems 46 (2015), 36-50.

Amazon Web Services. 2015a. Amazon EC2. Online article. (2015). http://aws.amazon.com/ec2/

Amazon Web Services. 2015b. Amazon EC2 Container Registry. (2015). https://aws.amazon.com/ecr/

Amazon Web Services. 2015c. AWS Management Console. (2015). https://aws.amazon.com/console/

shipyard. 2015. Shipyard Walkthrough. Online article. (2015). Retrieved November 10, 2015 from https: //shipyard-project.com/walkthrough/

Junaid Shuja, Kashif Bilal, Sajjad A Madani, Mazliza Othman, Rajiv Ranjan, Pavan Balaji, and Samee U Khan. 2014. Survey of techniques and architectures for designing energy-efficient data centers. (2014).

Sukhpal Singh and Inderveer Chana. 2015. QoS-Aware Autonomic Resource Management in Cloud Computing: A Systematic Review. ACM Comput. Surv. 48, 3, Article 42 (Dec. 2015), 46 pages.

Sukhpal Singh and Inderveer Chana. 2016. QoS-aware autonomic resource management in cloud computing: a systematic review. ACM Computing Surveys (CSUR) 48, 3 (2016), 42.

James Skene, Franco Raimondi, and Wolfgang Emmerich. 2010. Service-level agreements for electronic services. Software Engineering, IEEE Transactions on 36, 2 (2010), 288-304.

M. Smit, B. Simmons, M. Shtern, and M. Litoiu. 2013. Supporting application development with structured queries in the cloud. In Software Engineering (ICSE), 2013 35th International Conference on. 12131216. DOI : http://dx.doi.org/10.1109/ICSE.2013.6606681

StackEngine. 2015. StackEngine Container Application Center. (2015). http://stackengine.com/product/

CA Technologies. 2013. INSSLR2 - Redundant HTTP Input Gateway with SSL Support. Online article. (2013). Retrieved July 10, 2015 from https://support.ca.com/cadocs/0/CA\%20AppLogic\%203\%208-ENU/ Bookshelf_Files/HTML/AppLogicDoc/index.htm?toc.htm?CatGatewayINSSLR2.html

R.W. Thrash. 2010. Building a Cloud Computing Specification: Fundamental Engineering for Optimizing Cloud Computing Initiatives. http://assets1.csc.com/innovation/downloads/CSC_Papers_2010_Building_ a_Cloud_Computing_Specification.pdf. (March 2010).

Doug Tidwell. 2009. The Simple Cloud API : Writing portable, interoperable applications for the cloud. (2009). http://www.ibm.com/developerworks/library/os-simplecloud/

Adel Nadjaran Toosi, Rodrigo N Calheiros, and Rajkumar Buyya. 2014. Interconnected cloud computing environments: Challenges, taxonomy, and survey. ACM Computing Surveys (CSUR) 47, 1 (2014), 7.

James Turnbull. 2014. The Docker Book: Containerization is the new virtualization. James Turnbull.

Ubuntu. 2013. Juju. Online article. (2013). http://www.ubuntu.com/cloud/tools/juju

Peter Van Roy and others. 2009. Programming paradigms for dummies: What every programmer should know. New computational paradigms for computer music 104 (2009).

David Villegas and et al. 2012. Cloud Federation in a Layered Service Model. J. Comput. Syst. Sci. 78, 5 (Sept. 2012), 1330-1344. DOI : http://dx.doi.org/10.1016/j.jcss.2011.12.017

VisualOps. 2015. VisualOps - WYSIWYG for your cloud. Online article. (2015). Retrieved June 8, 2015 from http://docs.visualops.io/

Inc. VMware. 2015. Understanding virtual machine snapshots in VMware ESXi and ESX (1015180). Online article. (2015). Retrieved November 17, 2015 from http://kb.vmware.com/selfservice/microsites/search. do?language $=$ en_US\&cmd=displayKC\&externalId $=1015180$

Lizhe Wang, Rajiv Ranjan, Jinjun Chen, and Boualem Benatallah. 2012. Cloud computing: methodology, systems, and applications. CRC Press. 
Denis Weerasiri, Moshe Chai Barukh, Boualem Benatallah, and Cao Jian. 2016. CloudMap: A Visual Notation for Representing and Managing Cloud Resources. In International Conference on Advanced Information Systems Engineering. Springer, 427-443.

Denis Weerasiri and Boualem Benatallah. 2015. Unified Representation and Reuse of Federated Cloud Resources Configuration Knowledge. In Enterprise Distributed Object Computing Conference (EDOC), 2015 IEEE 19th International. 142-150.

Denis Weerasiri, Boualem Benatallah, and Moshe Chai Barukh. 2015a. Process-driven Configuration of Federated Cloud Resources. In Database Systems for Advanced Applications. Springer, 334-350.

Denis Weerasiri, Boualem Benatallah, and Moshe Chai Barukh. 2015b. ProcessBase: a hybrid process management platform. In Submitted to Service-Oriented Computing.

Yi Wei and M Brian Blake. 2013. Adaptive Service Workflow Configuration and Agent-Based Virtual Resource Management in the Cloud*. In Cloud Engineering (IC2E), 2013 IEEE International Conference on. IEEE, 279-284.

Johannes Wettinger, Uwe Breitenbücher, and Frank Leymann. 2014. Standards-based DevOps Automation and Integration Using TOSCA. In Proceedings of the 2014 IEEE / ACM 7th International Conference on Utility and Cloud Computing. IEEE Computer Society, 59-68.

Johannes Wettinger and et al. 2014. Unified Invocation of Scripts and Services for Provisioning, Deployment, and Management of Cloud Applications Based on TOSCA. In CLOSER 2014, April 3-5, 2014. SciTePress, 559-568.

Matthew S. Wilson. 2009. Constructing and Managing Appliances for Cloud Deployments from Repositories of Reusable Components. In Proceedings of the 2009 Conference on HotCloud'09. USENIX Association.

Erik Wittern, Alexander Lenk, Sebastian Bartenbach, and Tobias Braeuer. 2014. Feature-based Configuration of Vendor-independent Deployments on IaaS. In Enterprise Distributed Object Computing Conference (EDOC), 2014 IEEE 18th International. IEEE, 128-135.

Cheng-Zhong Xu, Jia Rao, and Xiangping Bu. 2012. URL: A unified reinforcement learning approach for autonomic cloud management. J. Parallel and Distrib. Comput. 72, 2 (2012), 95-105.

Eric Yuan, Naeem Esfahani, and Sam Malek. 2014. A systematic survey of self-protecting software systems. ACM Transactions on Autonomous and Adaptive Systems (TAAS) 8, 4 (2014), 17.

Rostyslav Zabolotnyi, Philipp Leitner, and Schahram Dustdar. 2014. Profiling-Based Task Scheduling for Factory-Worker Applications in Infrastructure-as-a-Service Clouds. In Software Engineering and Advanced Applications (SEAA), 2014 40th EUROMICRO Conference on. IEEE, 119-126.

Rostyslav Zabolotnyi, Philipp Leitner, Stefan Schulte, and Schahram Dustdar. 2015. SPEEDL - A Declarative Event-Based Language to Define the Scaling Behavior of Cloud Applications. In Services (SERVICES), 2015 IEEE World Congress on. 71-78. DOI : http://dx.doi.org/10.1109/SERVICES.2015.19

Peter Zadrozny and Raghu Kodali. 2013. Big Data Analytics Using Splunk: Deriving Operational Intelligence from Social Media, Machine Data, Existing Data Warehouses, and Other Real-Time Streaming Sources.

Liangzhao Zeng, B. Benatallah, A.H.H. Ngu, M. Dumas, J. Kalagnanam, and H. Chang. 2004. QoS-aware middleware for Web services composition. Software Engineering, IEEE Transactions on 30, 5 (May 2004), 311-327. DOI: http://dx.doi.org/10.1109/TSE.2004.11

Zhi-Hui Zhan, Xiao-Fang Liu, Yue-Jiao Gong, Jun Zhang, Henry Shu-Hung Chung, and Yun Li. 2015a. Cloud Computing Resource Scheduling and a Survey of Its Evolutionary Approaches. ACM Comput. Surv. 47, 4, Article 63 (July 2015), 33 pages. DOI : http://dx.doi.org/10.1145/2788397

Zhi-Hui Zhan, Xiao-Fang Liu, Yue-Jiao Gong, Jun Zhang, Henry Shu-Hung Chung, and Yun Li. 2015b. Cloud computing resource scheduling and a survey of its evolutionary approaches. ACM Computing Surveys (CSUR) 47, 4 (2015), 63.

Miranda Zhang, Rajiv Ranjan, Armin Haller, Dimitrios Georgakopoulos, Michael Menzel, and Surya Nepal. 2012b. An ontology-based system for Cloud infrastructure services' discovery. In Collaborative Computing: Networking, Applications and Worksharing (CollaborateCom), International Conference on. IEEE.

Miranda Zhang, Rajiv Ranjan, Anna Haller, Dimitrios Georgakopoulos, and Peter Strazdins. 2012a. Investigating decision support techniques for automating cloud service selection. In Cloud Computing Technology and Science (CloudCom), 2012 IEEE 4th International Conference on. IEEE, 759-764.

Miranda Zhang, Rajiv Ranjan, Surya Nepal, Michael Menzel, and Armin Haller. 2012c. A Declarative Recommender System for Cloud Infrastructure Services Selection. In Proceedings of the 9th International Conference on Economics of Grids, Clouds, Systems, and Services (GECON'12). Springer-Verlag, Berlin, Heidelberg, 102-113. DOI : http://dx.doi.org/10.1007/978-3-642-35194-5_8

Xinwen Zhang, Anugeetha Kunjithapatham, Sangoh Jeong, and Simon Gibbs. 2011. Towards an elastic application model for augmenting the computing capabilities of mobile devices with cloud computing. Mobile Networks and Applications 16, 3 (2011), 270-284. 


\section{Online Appendix to: A Taxonomy and Survey of Cloud Resource Orchestration Techniques}

DENIS WEERASIRI, University of New South Wales, Australia MOSHE CHAI BARUKH, University of New South Wales, Australia BOUALEM BENATALLAH, University of New South Wales, Australia QUAN Z. SHENG, University of Adelaide, Australia RAJIV RANJAN, University of Newcastle, United Kingdom

\section{A. LIST OF REFERENCES ORGANIZED BY TAXONOMY}

In this section, we list the name (if any) and reference of the methods, techniques and tools described along this work identifying the characteristic/s for which they were included.

\section{A.1. Resources and User Type}

Table V summarizes the examples used to illustrate the different dimensions of Resources and User Types.

Table V: Representative literature references for the Resources and User Types dimensions

\begin{tabular}{|c|c|c|}
\hline & Types & References \\
\hline \multirow[t]{3}{*}{ Resource Types } & Infrastructure & $\begin{array}{l}\text { AWS CloudFormation [Amazon 2011] } \\
\text { VMWare vSphere [Lowe 2011] } \\
\text { Juju [Ubuntu 2013] } \\
\text { Google Cloud (https://cloud.google.com/) } \\
\text { CohesiveFT [Networks 2016] } \\
\text { OpenNebula [Project 2016] } \\
\text { Eucalyptus [Development 2016] } \\
\text { Nectar [Directorate 2016] } \\
\text { Apache CloudStack [CloudStack 2016] } \\
\text { OpenTOSCA [Binz and et al. 2013] }\end{array}$ \\
\hline & Platform & $\begin{array}{l}\text { AWS OpsWorks [Rosner 2013] } \\
\text { AWS CloudFormation [Amazon 2011] } \\
\text { Heroku [Middleton et al. 2013] } \\
\text { terminal (https:///www.terminal.com/) } \\
\text { Puppet [Kanies 2006] } \\
\text { Juju [Ubuntu 2013] } \\
\text { Docker [Turnbull 2014] } \\
\text { OpenTOSCA [Binz and et al. 2013] } \\
\text { CFEngine [Burgess and College 1995] } \\
\text { Plush [Albrecht and et al. 2011] } \\
\text { SmartFrog [Goldsack and at al. 2009] } \\
\text { nitrous.io [Nitrous 2013] } \\
\text { Chef (https://www.chef.io/) } \\
\text { Ansible [Mohaan and Raithatha 2014] } \\
\text { RightScale [Adler 2011] } \\
\text { VisualOps [VisualOps 2015] } \\
\text { Skyport [Gerlach and et al. 2014] } \\
\text { EngineYard [Engine Yard 2016] } \\
\text { CloudBees [CloudBees 2016] } \\
\text { SaltStack [Hosmer 2012] }\end{array}$ \\
\hline & Software & Salesforce (http://www.salesforce.com) \\
\hline
\end{tabular}

(C) 2016 ACM 1539-9087/2016/06-ART0 $\$ 15.00$

DOI : http://dx.doi.org/10.1145/0000000.0000000 
Table V - Continued from previous page

\begin{tabular}{|c|c|c|}
\hline \multicolumn{2}{|c|}{ Resources and User Types } & \multirow[t]{2}{*}{ References } \\
\hline Resource Entity Model & $\begin{array}{l}\text { Applicable for any cloud } \\
\text { resource orchestration } \\
\text { technique }\end{array}$ & \\
\hline \multirow[t]{4}{*}{ Resource Access Methods } & CLIs & $\begin{array}{l}\text { AWS OpsWorks [Rosner 2013] } \\
\text { AWS CloudFormation [Amazon 2011] } \\
\text { Rackspace [Cali 2013] } \\
\text { VMWare vSphere [Lowe 2011] } \\
\text { Heroku [Middleton et al. 2013] } \\
\text { Puppet [Kanies 2006] } \\
\text { Juju [Ubuntu 2013] } \\
\text { Docker [Turnbull 2014] } \\
\text { CFEngine [Burgess and College 1995] } \\
\text { Plush [Albrecht and et al. 2011] } \\
\text { SmartFrog [Goldsack and at al. 2009] } \\
\text { Chef (https://www.chef.io/) } \\
\text { Ansible [Mohaan and Raithatha 2014] } \\
\text { SaltStack [Hosmer 2012] }\end{array}$ \\
\hline & SDKs & $\begin{array}{l}\text { AWS OpsWorks [Rosner 2013] } \\
\text { AWS Java SDK [Amazon Web Services 2015c] } \\
\text { Rackspace (https://developer.rackspace.com/sdks/) } \\
\text { jCloud [Foundation 2014c] } \\
\text { SimpleCloud [Tidwell 2009] } \\
\text { DeltaCloud [Foundation 2014a] } \\
\text { LibCloud [Foundation 2015c] }\end{array}$ \\
\hline & APIs & $\begin{array}{l}\text { AWS OpsWorks [Rosner 2013] } \\
\text { AWS CloudFormation [Amazon 2011] } \\
\text { AWS REST API for S3 [Amazon Web Services 2015d] } \\
\text { Rackspace (http://docs.rackspace.com/) } \\
\text { VMWare vSphere [Lowe 2011] } \\
\text { Heroku [Middleton et al. 2013] } \\
\text { Puppet [Kanies 2006] } \\
\text { Juju [Ubuntu 2013] } \\
\text { Docker [Turnbull 2014] } \\
\text { Chef (https://www.chef.io/) } \\
\text { Ansible [Mohaan and Raithatha 2014] } \\
\text { SaltStack [Hosmer 2012] }\end{array}$ \\
\hline & GUIs & $\begin{array}{l}\text { AWS OpsWorks [Rosner 2013] } \\
\text { VMWare vSphere [Lowe 2011] } \\
\text { Puppet [Kanies 2006] } \\
\text { Juju-GUI (https://demo.jujucharms.com/) } \\
\text { OpenTOSCA [Binz and et al. 2013] } \\
\text { VisualOps [VisualOps 2015] } \\
\text { AWS Management Console [Services 2015c] } \\
\text { Puppet Management Console [Labs 2015c] } \\
\text { CA-Applogic [AppLogic 2015] } \\
\text { StackEngine [StackEngine 2015] } \\
\text { Panamax [CenturyLink 2015] } \\
\text { Shipyard [shipyard 2015] } \\
\text { CFEngine [Burgess and College 1995] }\end{array}$ \\
\hline Resource Representation & $\begin{array}{l}\text { Textual } \\
\text { n }\end{array}$ & $\begin{array}{l}\text { AWS OpsWorks [Rosner 2013] } \\
\text { AWS CloudFormation [Amazon 2011] } \\
\text { Puppet [Kanies 2006] } \\
\text { Juju [Ubuntu 2013] } \\
\text { Docker [Turnbull 2014] } \\
\text { OpenTOSCA [Binz and et al. 2013] } \\
\text { CFEngine [Burgess and College 1995] } \\
\text { Plush [Albrecht and et al. 2011] } \\
\text { SmartFrog [Goldsack and at al. 2009] } \\
\text { Chef (https://www.chef.io/) } \\
\text { Ansible [Mohaan and Raithatha 2014] } \\
\text { Docker Compose [Docker 2015b] } \\
\text { AWS CLI [AWS 2013b] } \\
\text { SaltStack [Hosmer 2012] }\end{array}$ \\
\hline
\end{tabular}


Table V - Continued from previous page

\begin{tabular}{|c|c|c|}
\hline & Resources and User Types & References \\
\hline & Visual & $\begin{array}{l}\text { Cloud Computing Patterns [Leymann et al. 2014] } \\
\text { AWS OpsWorks [Rosner 2013] } \\
\text { Puppet [Kanies 2006] } \\
\text { Juju [Ubuntu 2013] } \\
\text { OpenTOSCA [Binz and et al. 2013] } \\
\text { CFEngine [Burgess and College 1995] } \\
\text { nitrous.io [Nitrous 2013] } \\
\text { Chef (https://www.chef.io/) } \\
\text { Ansible [Mohaan and Raithatha 2014] } \\
\text { VisualOps [VisualOps 2015] } \\
\text { Hyperglance [Ltd 2015] } \\
\text { RightScale [Adler 2011] }\end{array}$ \\
\hline & Hybrid & $\begin{array}{l}\text { AWS OpsWorks [Rosner 2013] } \\
\text { Juju [Ubuntu 2013] } \\
\text { OpenTOSCA [Binz and et al. 2013] }\end{array}$ \\
\hline \multirow[t]{3}{*}{ User Types } & DevOps & $\begin{array}{l}\text { Google Cloud (https://cloud.google.com/) } \\
\text { AWS OpsWorks [Rosner 2013] } \\
\text { AWS CloudFormation [Amazon 2011] } \\
\text { VMWare vSphere [Lowe 2011] } \\
\text { Puppet [Kanies 2006] } \\
\text { Juju [Ubuntu 2013] } \\
\text { Docker [Turnbull 2014] } \\
\text { OpenTOSCA [Binz and et al. 2013] } \\
\text { CFEngine [Burgess and College 1995] } \\
\text { Plush [Albrecht and et al. 2011] } \\
\text { SmartFrog [Goldsack and at al. 2009] } \\
\text { Chef (https://www.chef.io/) } \\
\text { Ansible [Mohaan and Raithatha 2014] } \\
\text { RightScale [Adler 2011] } \\
\text { VisualOps [VisualOps 2015] } \\
\text { SaltStack [Hosmer 2012] }\end{array}$ \\
\hline & $\begin{array}{l}\text { Application De- } \\
\text { velopers }\end{array}$ & $\begin{array}{l}\text { Heroku [Middleton et al. 2013] } \\
\text { terminal (https://www.terminal.com/) } \\
\text { nitrous.io [Nitrous 2013] }\end{array}$ \\
\hline & Domain Experts & $\begin{array}{l}\text { Skyport [Gerlach and et al. 2014] } \\
\text { CS50 appliance [CS50 2015] }\end{array}$ \\
\hline
\end{tabular}

\section{A.2. Resource Orchestration Capabilities}

Table VI summarizes the examples used to illustrate the different dimensions of Resource Orchestration Capabilities.

Table VI: Representative literature references for the Resource Orchestration Capabilities dimension

\begin{tabular}{ll}
\hline \multicolumn{1}{c}{ Resource Orchestration Capabilities } & \multicolumn{1}{c}{ References } \\
\hline & \\
& AWS Marketplace [Marketplace 2012] \\
& Bitnami [Bitnami 2015] \\
& Puppet Forge (https://forge.puppetlabs.com/) \\
& Docker Hub Registry [Docker 2015a] \\
& [Weerasiri and Benatallah 2015] \\
Primitive Actions & [Zhang et al. 2012c] \\
& Juju Charms (https://jujucharms.com/store) \\
& Terminal.com (https://www.terminal.com/explore) \\
&
\end{tabular}


Table VI - Continued from previous page

\begin{tabular}{|c|c|c|}
\hline \multicolumn{2}{|c|}{ Resource Orchestration Capabilities } & References \\
\hline & Configure & $\begin{array}{l}\text { Puppet [Kanies 2006] } \\
\text { Chef (https://www.chef.io/) } \\
\text { Ansible [Mohaan and Raithatha 2014] } \\
\text { CFEngine [Burgess and College 1995] } \\
\text { VisualOps [VisualOps 2015] } \\
\text { [Kirschnick and et al. 2012] } \\
\text { SaltStack [Hosmer 2012] }\end{array}$ \\
\hline & Deploy & $\begin{array}{l}\text { Puppet [Kanies 2006] } \\
\text { Juju [Ubuntu 2013] } \\
\text { Docker [Turnbull 2014] } \\
\text { OpenTOSCA [Binz and et al. 2013] } \\
\text { CFEngine [Burgess and College 1995] } \\
\text { Plush [Albrecht and et al. 2011] } \\
\text { SmartFrog [Goldsack and at al. 2009] } \\
\text { Chef (https://www.chef.io/) } \\
\text { Ansible [Mohaan and Raithatha 2014] } \\
\text { [Kirschnick and et al. 2012] } \\
\text { AWS OpsWorks [Rosner 2013] } \\
\text { AWS CloudFormation [Amazon 2011] } \\
\text { VisualOps [VisualOps 2015] } \\
\text { [Weerasiri et al. 2015a] } \\
\text { [Wettinger and et al. 2014] }\end{array}$ \\
\hline & Monitor & $\begin{array}{l}\text { Nagios [Barth 2008] } \\
\text { AWS CloudWatch [CloudWatch 2013] } \\
\text { AWS CloudTrail [CloudTrail 2014] } \\
\text { CloudFielder [MadeiraCloud 2015] } \\
\text { Splunk [Zadrozny and Kodali 2013] } \\
\text { Finally.io [Finally.io 2014] }\end{array}$ \\
\hline & Control & $\begin{array}{l}\text { AWS OpsWorks [Rosner 2013] } \\
\text { Juju [Ubuntu 2013] } \\
\text { [Weerasiri et al. 2015a] } \\
\text { Finally.io [Finally.io 2014] }\end{array}$ \\
\hline \multirow[t]{3}{*}{ Orchestration Strategies } & User-defined & $\begin{array}{l}\text { Docker [Turnbull 2014] } \\
\text { [Wettinger and et al. 2014] } \\
\text { [Liu et al. 2011a] } \\
\text { [Ranjan et al. 2015] } \\
\text { [Lu et al. 2013] } \\
\text { [Zeng et al. 2004] } \\
\text { [Lu et al. 2013] }\end{array}$ \\
\hline & Rule-based & $\begin{array}{l}\text { Juju [Ubuntu 2013] } \\
\text { AWS OpsWorks [Rosner 2013] } \\
\text { CloudFielder [MadeiraCloud 2015] } \\
\text { [Chapman et al. 2012] } \\
\text { [Zhang et al. 2011] } \\
\text { [Zabolotnyi et al. 2015] } \\
\text { [Weerasiri et al. 2015a] }\end{array}$ \\
\hline & $\begin{array}{l}\text { State Machine- } \\
\text { based }\end{array}$ & TIBCO [Inc. 2014] \\
\hline
\end{tabular}


Table VI - Continued from previous page

\begin{tabular}{|c|c|c|}
\hline \multicolumn{2}{|c|}{ Resource Orchestration Capabilities } & \multirow[b]{2}{*}{$\begin{array}{l}\text { [Schulte et al. 2015] } \\
\text { [Wei and Blake 2013] } \\
\text { [Zhan et al. 2015a] } \\
\text { [Singh and Chana 2015] } \\
\text { [Menzel et al. 2015] } \\
\text { [Zhang et al. 2012a] } \\
\text { [Gravier et al. 2015] } \\
\text { [Xu et al. 2012] } \\
\text { [Ding et al. 2014] } \\
\text { [Ramezani et al. 2013] } \\
\text { [Islam et al. 2012] } \\
\text { [Antonescu et al. 2013] } \\
\text { [Fang et al. 2012] } \\
\text { [Jiang et al. 2011] } \\
\text { [Pandey et al. 2010] } \\
\text { [Iqbal et al. 2011] } \\
\text { [Beloglazov et al. 2012] }\end{array}$} \\
\hline & Autonomic & \\
\hline \multirow[t]{6}{*}{ Language Paradigm } & Script-based & $\begin{array}{l}\text { AWS OpsWorks [Rosner 2013] } \\
\text { Juju [Ubuntu 2013] } \\
\text { Docker [Turnbull 2014] } \\
\text { Plush [Albrecht and et al. 2011] } \\
\text { SmartFrog [Goldsack and at al. 2009] } \\
\text { Chef (https://www.chef.io/) } \\
\text { Ansible [Mohaan and Raithatha 2014] } \\
\text { Puppet [Kanies 2006] } \\
\text { SaltStack [Hosmer 2012] }\end{array}$ \\
\hline & Flow-based & $\begin{array}{l}\text { [Kopp and et al. 2012] } \\
\text { [Weerasiri et al. 2015a] }\end{array}$ \\
\hline & ECA rule-based & $\begin{array}{l}\text { AWS OpsWorks [Rosner 2013] } \\
\text { CloudFielder [MadeiraCloud 2015] } \\
\text { Juju [Ubuntu 2013] }\end{array}$ \\
\hline & $\begin{array}{l}\text { Markup lan- } \\
\text { guages }\end{array}$ & $\begin{array}{l}\text { Plush [Albrecht and et al. 2011] } \\
\text { AWS CloudFormation [Amazon 2011] } \\
\text { DotCloud [dotCloud 2015] }\end{array}$ \\
\hline & Query-based & $\begin{array}{l}\text { [Liu et al. 2011a] } \\
\text { [Lu et al. 2013] }\end{array}$ \\
\hline & $\begin{array}{l}\text { Constraint pro- } \\
\text { gramming }\end{array}$ & $\begin{array}{l}\text { CFEngine [Burgess and College 1995] } \\
\text { [Danninger 2015] } \\
\text { [Sawyer et al. 2012] }\end{array}$ \\
\hline Theoretical Foundation & Formal methods & $\begin{array}{l}\text { [Burgess and Couch 2006] } \\
\text { [Bergstra and Burgess 2014] } \\
\text { [Burgess and College 1995] } \\
\text { [Couch et al. 2003] } \\
\text { [Burgess 2011] } \\
\text { [Burgess 2009] } \\
\text { [Burgess 2007] } \\
\text { [Borril et al. 2014] }\end{array}$ \\
\hline \multirow{3}{*}{ Cross-cutting Concerns } & Security & $\begin{array}{l}\text { [Fritsch 2015] } \\
\text { [Sato et al. 2010] }\end{array}$ \\
\hline & SLAs & $\begin{array}{l}\text { [Gravier and et al. 2013] } \\
\text { [Skene et al. 2010] } \\
\text { [Ludwig et al. 2003] }\end{array}$ \\
\hline & Portability & $\begin{array}{l}\text { [Lewis et al. 2013] } \\
\text { [OASIS 2013] } \\
\text { [Turnbull 2014] } \\
\text { [OpenStack.org 2015a] } \\
\text { [Foundation 2015a] }\end{array}$ \\
\hline
\end{tabular}




\section{A.3. Knowledge Reuse}

Table VII summarizes the examples used to illustrate the different dimensions of Knowledge Reuse.

Table VII: Representative literature references for the Knowledge Reuse dimension

\begin{tabular}{|c|c|c|}
\hline \multicolumn{2}{|c|}{ Knowledge Reuse } & References \\
\hline \multirow[t]{3}{*}{ Reuse Artifact } & $\begin{array}{l}\text { Concrete and Tem- } \\
\text { plate resource de- } \\
\text { scriptions }\end{array}$ & $\begin{array}{l}\text { AWS OpsWorks [Rosner 2013] } \\
\text { AWS CloudFormation [Amazon 2011] } \\
\text { Heroku [Middleton et al. 2013] } \\
\text { Puppet [Kanies 2006] } \\
\text { Juju [Ubuntu 2013] } \\
\text { Docker [Turnbull 2014] } \\
\text { OpenTOSCA [Binz and et al. 2013] } \\
\text { CFEngine [Burgess and College 1995] } \\
\text { Plush [Albrecht and et al. 2011] } \\
\text { SmartFrog [Goldsack and at al. 2009] } \\
\text { Chef (https://www.chef.io/) } \\
\text { Ansible [Mohaan and Raithatha 2014] } \\
\text { RightScale [Adler 2011] } \\
\text { SaltStack [Hosmer 2012] }\end{array}$ \\
\hline & $\begin{array}{l}\text { Resource snap- } \\
\text { shots }\end{array}$ & $\begin{array}{l}\text { VMWare vSphere [Lowe 2011; VMware 2015] } \\
\text { terminal (https://www.terminal.com/) [Inc. 2015] }\end{array}$ \\
\hline & Miscellaneous & $\begin{array}{l}\text { Juju [Ubuntu 2013] } \\
\text { Docker [Turnbull 2014] }\end{array}$ \\
\hline \multirow{3}{*}{$\begin{array}{l}\text { [Zhang et al. } \\
\text { 2012c] } \\
\text { [Weerasiri and } \\
\text { Benatallah } \\
\text { 2015] }\end{array}$} & Search index & $\begin{array}{l}\text { Bitnami [Bitnami 2015] } \\
\text { VMWare vSphere [Lowe 2011] } \\
\text { AWS OpsWorks [Rosner 2013] } \\
\text { AWS CloudFormation [Amazon 2011] } \\
\text { Puppet [Kanies 2006] } \\
\text { Juju [Ubuntu 2013] } \\
\text { Docker [Turnbull 2014] } \\
\text { CFEngine [Burgess and College 1995] } \\
\text { Chef (https://www.chef.io/) } \\
\text { Ansible [Mohaan and Raithatha 2014] } \\
\text { VisualOps [VisualOps 2015] } \\
\text { RightScale [Adler 2011] }\end{array}$ \\
\hline & Recommendations & AWS Marketplace [Marketplace 2012] \\
\hline & Community-based & $\begin{array}{l}\text { Puppet [Kanies 2006] } \\
\text { Juju [Ubuntu 2013] } \\
\text { Docker [Turnbull 2014] } \\
\text { Chef (https://www.chef.io/) } \\
\text { Ansible [Mohaan and Raithatha 2014] }\end{array}$ \\
\hline
\end{tabular}

\section{A.4. Runtime Environment}

Table VIII summarizes the examples used to illustrate the different dimensions of Runtime Environment. 
Table VIII: Representative literature references for the Runtime Environment dimension

\begin{tabular}{|c|c|c|}
\hline \multicolumn{2}{|c|}{ Runtime Environment } & References \\
\hline \multirow[t]{2}{*}{ Virtualization Technique } & OS-level hypervisor & $\begin{array}{l}\text { VMWare vSphere [Lowe 2011] } \\
\text { Google Cloud (https://cloud.google.com/) } \\
\text { AWS OpsWorks [Rosner 2013] } \\
\text { AWS CloudFormation [Amazon 2011] } \\
\text { Juju [Ubuntu 2013] } \\
\text { OpenTOSCA [Binz and et al. 2013] }\end{array}$ \\
\hline & $\begin{array}{l}\text { Environment- } \\
\text { level Container } \\
\text { manager }\end{array}$ & $\begin{array}{l}\text { Heroku [Middleton et al. 2013] } \\
\text { Skyport [Gerlach and et al. 2014] } \\
\text { Docker [Turnbull 2014] }\end{array}$ \\
\hline \multirow[t]{2}{*}{ Execution Model } & Centralized & $\begin{array}{l}\text { VMWare vSphere [Lowe 2011] } \\
\text { AWS OpsWorks [Rosner 2013] } \\
\text { AWS CloudFormation [Amazon 2011] } \\
\text { Heroku [Middleton et al. 2013] } \\
\text { Juju [Ubuntu 2013] } \\
\text { Docker [Turnbull 2014] } \\
\text { OpenTOSCA [Binz and et al. 2013] } \\
\text { Plush [Albrecht and et al. 2011] } \\
\text { SmartFrog [Goldsack and at al. 2009] } \\
\text { Chef (https://www.chef.io/) } \\
\text { Ansible [Mohaan and Raithatha 2014] } \\
\text { SaltStack [Hosmer 2012] }\end{array}$ \\
\hline & De-centralized & $\begin{array}{l}\text { Puppet [Kanies 2006] } \\
\text { Skyport [Gerlach and et al. 2014] } \\
\text { [Kirschnick and et al. 2012] } \\
\text { CFEngine [Burgess and College 1995] }\end{array}$ \\
\hline \multirow[t]{2}{*}{ Target Environment } & Public & $\begin{array}{l}\text { Google Cloud (https://cloud.google.com/) } \\
\text { Terminal (https://www.terminal.com/) } \\
\text { Nitrous.io [Nitrous 2013] } \\
\text { Chef (https://www.chef.io/) } \\
\text { Ansible [Mohaan and Raithatha 2014] } \\
\text { RightScale [Adler 2011] } \\
\text { SaltStack [Hosmer 2012] } \\
\text { ElasticBox (https://elasticbox.com/) } \\
\text { AWS OpsWorks [Rosner 2013] } \\
\text { AWS CloudFormation [Amazon 2011] } \\
\text { Heroku [Middleton et al. 2013] } \\
\text { Puppet [Kanies 2006] } \\
\text { Juju [Ubuntu 2013] } \\
\text { Docker [Turnbull 2014] } \\
\text { OpenTOSCA [Binz and et al. 2013] } \\
\text { VisualOps [VisualOps 2015] } \\
\text { CFEngine [Burgess and College 1995] }\end{array}$ \\
\hline & Private & $\begin{array}{l}\text { Chef (https://www.chef.io/) } \\
\text { Ansible [Mohaan and Raithatha 2014] } \\
\text { SaltStack [Hosmer 2012] } \\
\text { ElasticBox (https://elasticbox.com/) } \\
\text { VMWare vSphere [Lowe 2011] } \\
\text { Puppet [Kanies 2006] } \\
\text { Juju [Ubuntu 2013] } \\
\text { Docker [Turnbull 2014] } \\
\text { OpenTOSCA [Binz and et al. 2013] } \\
\text { CFEngine [Burgess and College 1995] } \\
\text { Plush [Albrecht and et al. 2011] } \\
\text { SmartFrog [Goldsack and at al. 2009] }\end{array}$ \\
\hline
\end{tabular}


Table VIII - Continued from previous page

\begin{tabular}{ll}
\hline Runtime Environment & \multicolumn{1}{c}{ References } \\
\hline & [Weerasiri et al. 2015a] \\
[Wettinger and et al. 2014] & [Wettinger et al. 2014] \\
[Weerasiri et al. 2015b] & TOSCA [OASIS 2013] \\
& OpenTOSCA [Binz and et al. 2013] \\
& [Moscato and et al. 2011] \\
& [Smit et al. 2013] \\
& jCloud [Foundation 2014c] \\
& SimpleCloud [Tidwell 2009] \\
& DeltaCloud [Foundation 2014a] \\
& Skyport [Gerlach and et al. 2014] \\
& LibCloud [Foundation 2015c] \\
& CohesiveFT [Networks 2016] \\
& OpenNebula [Project 2016] \\
& Eucalyptus [Development 2016] \\
& Cloudward Bound [Hajjat et al. 2011] \\
& Ansible Cloud Modules [Ansible 2015] \\
\hline
\end{tabular}

\section{B. CROSS-CUTTING CONCERNS}

Implementing cloud orchestration processes are met with a range of cross-cutting concerns, such as: security; service level agreements and negotiations; portability; interoperability; standardization; resource demand profiling; resource pricing; profit maximizing and other runtime issues. While we understand the importance of all this, we specifically focus on the orchestration aspects of cloud resources - in the sense of identifying abstractions to manage cloud resources. There are however other surveys with in-depth focus on these related aspects [Lewis et al. 2013; Huang and et al. 2015; Roy et al. 2015; Ardagna et al. 2015; Toosi et al. 2014; Jennings and Stadler 2014; Mann 2015]. 